\title{
A Modified ACO for Classification on different Data Set
}

\author{
Dharmpal Singh \\ Department of CSE \\ Pailan College of Management \\ and Technology \\ Sector - 1, Phase I, Bengal \\ Pailan Park, Off Diamond \\ Harbour Road, Joka, Kolkata, \\ West Bengal 700104
}

\author{
J. Paul Choudhury \\ Department of IT \\ Kalyani Govt. Engineering \\ College, Kalyani, Nadia- \\ 741235, West Bengal, INDIA
}

\author{
Mallika De \\ Department of Engineering \& \\ Technological Studies \\ (Retired), Kalyani \\ University Kalyani, Nadia- \\ 741235, West Bengal, INDIA
}

\begin{abstract}
Ant colony optimization algorithms have been applied to many combinatorial optimization problems, ranging from quadratic assignment to protein folding or routing vehicles and a lot of derived methods have been adapted to dynamic problems in real variables, stochastic problems, multi-targets and parallel implementations. It has also been used to classification of the data set based on the attribute. It has been observed that construct solution and pheromone update play an important role in the ACO algorithm. The selection of the pheromone update is based on the construct solution which is further base on the probability function and initial selection. So if the selection of the pheromone done properly then ACO algorithm will terminate in less number of the iteration and it will be produce the good result. It has further observed that difference result have been possible for the different selection of the construct and pheromone on the same data set. Therefore, in this paper an effort has been made to suggest the techniques to select the initial construct and pheromone update for data set and the classification has to be done using the concept of clustering.
\end{abstract}

\section{Keywords}

Data mining, soft computing, Ant colony optimization, Particle swarm optimization, fuzzy, neural network, data mining preprocessing.

\section{INTRODUCTION}

Ants live together in colonies and they use chemical cues called pheromones to provide a sophisticated communication system. An isolated ant moves essentially at random but an ant encountering a previously laid pheromone will detect it and decide to follow it with high probability and thereby reinforce it with a further quantity of pheromone. The repetition of the above mechanism represents the collective behaviour of a real ant colony which is a form of autocatalytic behaviour where the more the ants follow a trail, the more attractive that trail becomes. The above behaviour of real ants has inspired ACO which has proved to be an effective metaheuristic technique for solving many complex problems. This technique uses a colony of artificial ants that behaves as cooperative agents in a mathematical space where they are allowed to search and reinforce pathways (solutions) in order to find the optimal ones. The features of artificial ants are: having some memory, not being completely blind and the process time is discrete. In the ACO technique an initialisation phase takes place during which ants are positioned on different nodes (sessions) with empty tabu lists and initial pheromone distributed equally on paths connecting these sessions. Ants update the level of pheromone while they are constructing their schedules by iteratively adding new sessions to the current partial schedule. At each time step, ants compute a set of feasible moves and select the best one according to some probabilistic rules based on the heuristic information and pheromone level. The higher value of the pheromone and the heuristic information, the more profitable is to select this move and resume the search. The selected node is putted in the tabu list related to the ant to prevent to be chosen again. Heuristic information represents the nearer sessions around the current session, while pheromone level "memory" of each path represents the usability of this path in the past to find good schedules. At the end of each iteration, the tabu list for each ant will be full and the obtained cheapest schedule is computed and memorized. For the following iteration, tabu lists will be emptied ready for use and the pheromone level will be updated. This process is repeated till the number of iterations (stopping criteria) has been reached.

\subsection{ACO algorithm}

A general outline of the ACO algorithm is furnished below.

Algorithm ACO meta heuristic();

while (termination criterion not satisfied)

ant generation and activity();

pheromone evaporation();

daemon actions(); "optional"

end while

end Algorithm

\section{RELATED WORK AND LITERATURE SURVEY}

Thomas Stutzle and Marco Dorigo[1], have tried to solve the travelling salesman problem with an ant colony optimization and authors have given an overview on the available ACO algorithms for the TSP. Thereafter authors have outlined how ACO algorithms can be applied to TSP problem and presented the available ACO algorithms for the TSP. In section 1.4, they have briefly discusses local search for the TSP, while Section 1.5 presented experimental results which have been obtained with MAX -MIN Ant System. The authors have further opined that they have been applied to several other combinatorial optimization problems because it was the first application of ACO algorithms to the TSP. Furthermore, the authors opined that ACO algorithms have proved to be among the best available algorithms and in section 1.6 they have given a concise overview of these other applications of ACO algorithms. 
Wei Zhao et al.[2] have tried to solve combination optimization problems using ant colony optimization. The authors have presented revised pheromones in local and global update mode for solving TSP. The authors have proposed a pheromone increment model called ant constant and a pheromone diffusion model and shown that proposed model given better optimal solutions on different benchmark data sets.

Héctor D. Menéndez, et al. [3] have presented an ACObased clustering algorithm inspired by the ACO Clustering (ACOC) algorithm and authors have restructures ACOC from a centroid-based technique to a medoid-based technique, where the properties of the search space are not necessarily known. The authors have compared proposed algorithm with ACO Clustering (ACOC) algorithm on both synthetic datasets and real-world datasets extracted from the UCI Machine Learning Repository. The authors have opined that proposed algorithm outperformed the early algorithm based on its accuracy.

Xiaoyong Liu [4] has presented an improved clustering algorithm with Ant Colony optimization (ACO) based on dynamical pheromones. The author has proposed two strategies to improve the performance of the proposed algorithm. The author has adjusted the rate of pheromone evaporation dynamically, and the other has to adjust the strength of pheromone dynamically. The author has compared the proposed method based on the two indices named as Precision and Recall. The author has opined that it has given the best result as compared to the other algorithm.

R. F. Tavares Neto and M. Godinho Filho [13] have presented a literature survey on the uses of ACO approach to solve scheduling problems. The authors have opined that it will not only able to derive certain guidelines for the implementation of ACO algorithms but also to determine possible directions for future research.

Though the ant colony algorithms can resolve several optimization problems successfully, but it cannot establish its convergence. The many limitations have been observed from various survey paper which has been furnished below.

1) ACO has slow convergence speed and low efficiency $[7,8$, 10,11 , and 12].

2) It is likely to fall in the local optimal solution $[7,8,10,11$, and 12]. The main cause of this premature convergence is that the ant colony algorithms update the pheromone corresponding to the current better path, and after certain iterations, the pheromone on the best path becomes very strong, while the pheromone on the worthy path remains very weak. All the ants stick on the best path and it becomes very difficult to jump out from this best path. This raises the likelihood that the obtained optimal solution is only a local optimal solution.

3) Limited domain of inputs can be explored by using ACO $[5,10$, and 12]. The studies also reported that in those cases ACO could be applied only to integral input domain.

4) Some studies also stated that redundant paths could not be deleted by using the ACO technique [6,9]. This implies that various ants can find the test cases that meet the same test adequacy criterion and hence become redundant, whereas ants should be able to explore new paths without taking into account the redundant test cases.

5) Restricted applications of ACO were also spotted by a couple of studies $[10,12]$. According to these, their developed systems could not be useful for Object Oriented Programming. The reason behind this could be that the initial approach followed by them did not include the concept of object-oriented programming.

Several authors have used the ACO algorithm for the solving of TSP problem [1], combination optimization problems [2] and Clustering [3, 4]. Moreover authors have also pointed out the problem of ACO in slow convergence speed and low efficiency $[7,8,10,11$, and 12], fall in the local optimal solution [7, 8, 10, 11, and 12], limited domain of inputs can $[5,10$, and 12], redundant paths could not be deleted by using the ACO technique $[6,9]$ and restricted applications of ACO $[10,12]$. It has been observed that construct solution and pheromone update play an important role in the ACO algorithm. The selection of the pheromone update is based on the construct solution which is further base on the probability function and initial selection. So if the selection of the pheromone done properly then ACO algorithm will terminate in less number of the iteration and it will be produce the good result. It has further observed that difference result have been possible for the different selection of the construct and pheromone on the same data set. Therefore, in this paper an effort has been made to suggest the techniques to select the initial construct and pheromone update for data set such that it will produce same result for different data set. Here modified form of the ACO algorithm has also been proposed.

In first section, abstract and introduction have been furnished. In second section, related work and literature survey of the models have been furnished. In third section, implementation has been furnished in detail. In fourth section, result and conclusion have been furnished

\section{METHODOLOGY AND IMPLEMENTATION}

The concept of data mining [14], association rule [15], factor analysis [15], fuzzy logic $(14,1516)$ neural network [14], PSO [14] and ABC [17] have been elaborated in the mentioned paper. Therefore, due to size of paper only detail explanation of ACO has been furnished in section 3.1.

\subsection{Data cleaning and formation of association rule}

\subsubsection{Purpose of work}

The available data contains the information of Iris flowers with various items. The data related to iris flower containing 150 data values. It is to note that the quality of flower depends on the sepal length of the flower. If the sepal length of a particular flower is known, the quality of flower can be ascertained. Therefore the sepal length of flower (D) has been chosen as the objective item (consequent item) of the flowers. The other parameters i.e. sepal width (A), petal length (B) and petal width $(\mathrm{C})$ have been chosen as the depending items (antecedent items).

The purpose of this work is to correlate the items A, B and C with $D$, so that based on any value of $A, B$ and $C$, the value of $\mathrm{D}$ can be estimated. From the value of $\mathrm{D}$ the quality of that type of flower can be ascertained.

\subsubsection{Data mining Preprocessing}

Now it is necessary to check whether the data items are proper or not. If the data items are proper, extraction of information is possible otherwise the data items are not suitable for the extraction of knowledge. In that case preprocessing of data is 
necessary for getting the proper data. Therefore, the data mining preprocessing techniques like data cleansing, data integration, data transformation and data reduction have to be applied on the available data as follows:

\section{Data Cleansing}

The data cleansing techniques include the filling in the missing value, correct or rectify the inconsistent data and identify the outlier in the available data.

It has been observed that all the data sets which have been considered do not contain any missing value. The said data sets do not contain any inconsistent data i.e. any abnormally low or any abnormally high value. All the data values are regularly distributed within the range of that data items. Therefore the data cleansing techniques are not applicable for the available data.

\section{Data Integration}

The data integration technique has to be applied if data has been collected from different sources. The available data have been taken from a single source therefore the said technique is not applicable here.

\section{Data Transformation}

To make the data within specific range smoothing and normalization techniques have to be applied. Decimal scaling technique has to be applied to move the decimal point to particular position of all data values. Out of these data transformation, smoothing and decimal scaling (multiply by 1000) techniques have been applied on the set.

\section{Data Reduction}

The data reduction method has to be applied on huge amount of data (tetra byte) to get the certain portion of data. Since here the amount of data is not large, said technique has not been applied.

The data set has been taken from well know web site where it have been stored in well from Therefore, in this paper only data scaling has been applied on the said data set

\subsection{Implementation}

The details of the formation of association rule and application of the factor analysis and principal component analysis has been discussed in the paper [18]. The concept of data mining [14], association rule[15], factor analysis [15], fuzzy logic $(14,15$ 16) neural network [14], PSO [14] and $\mathrm{ABC}$ [17] have been elaborated in the mentioned paper. Due to size of the paper, only details procedure of ACO is discussed in this paper.

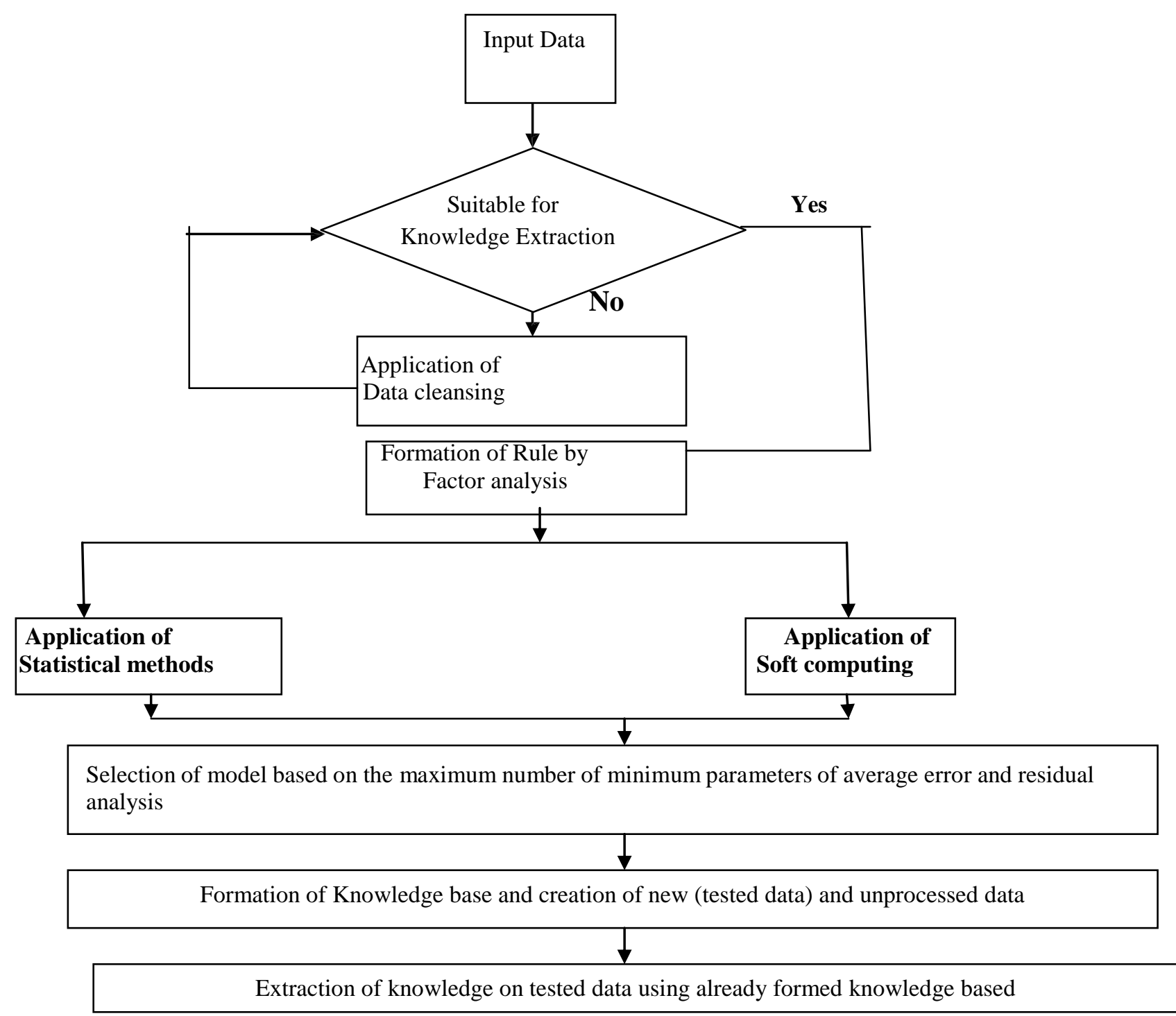




\subsection{Modified Ant Colony algorithm}

\section{Step 1}

Initialization of algorithm parameters

The universe of discourse $U$ has been chosen as minimum to maximum value and has been divided into six intervals. The intervals have been chosen as group one, group two, group three, group four, group five and group six respectively. These grouns have been further divided into six subgrouns.

\section{Step 2}

Calculation of relative distances

The relative distance of the input data value is taken as the result of the subtraction of input data value from middle value of its respective subgroups The relative distance value for the other elements from other subgroups has been calculated. and pheromone trials stored the error of the individual data with respect to middle value of the subgroup of data group

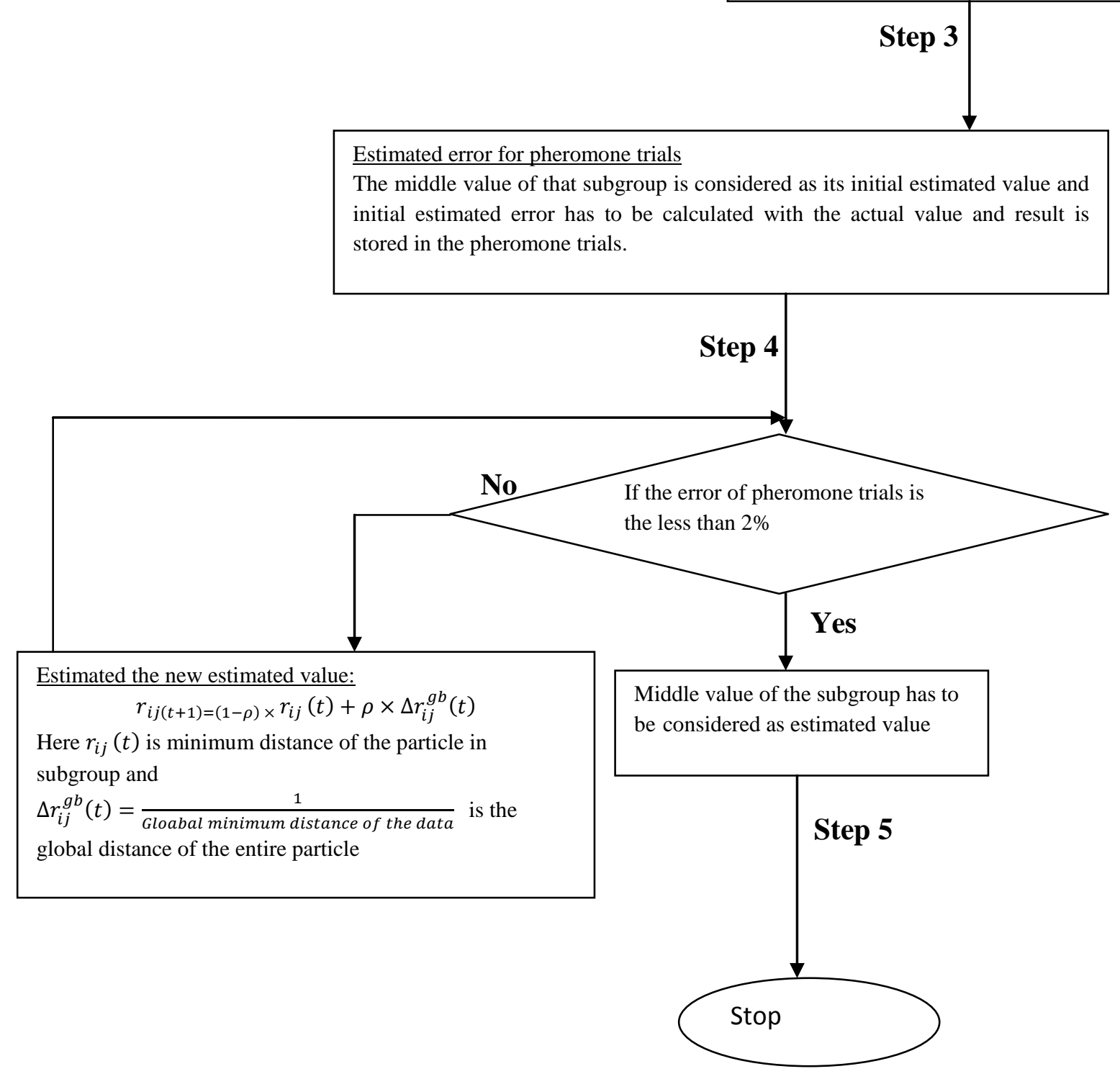




\subsection{Implementation Ant Colony Optimization}

The value encoding has to be considered as the data encoding and the (1/ estimated error) of the estimated data with the actual data has to be considered as a fitness function of the estimated data

The universe of discourse $\mathrm{U}$ has been chosen as 3330 to 9816 and has been divided into several intervals. The intervals have been chosen as $A_{1}, A_{2}, A_{3}, A_{4}, A_{5}$ and $A_{6}$ which are termed as group one, group two, group three, group four, group five and group six respectively. These groups have been further divided into six subgroups. The ranges of groups and subgroups have been furnished in table 1. Fuzzy sets have been defined on the universe and some linguistic values have also been determined. Let, $A_{1}=$ (many), $A_{2}=$ (many, many), $\mathrm{A}_{3}=$ (very many, $\mathrm{A}_{4}=$ (too many) be the possible values.

Table 1. Group and Subgroup Range

\begin{tabular}{|c|c|c|c|c|c|c|}
\hline $\begin{array}{c}\text { Group/Subgro } \\
\text { up }\end{array}$ & \multicolumn{6}{|c|}{ Subgroup } \\
\hline $\begin{array}{c}\text { Group One } \\
\left(\mathrm{A}_{1}\right)\end{array}$ & $\mathbf{A}_{11}$ & $\mathbf{A}_{12}$ & $A_{13}$ & $A_{14}$ & $\mathbf{A}_{15}$ & $A_{16}$ \\
\hline$(3300-4386)$ & $\begin{array}{l}(3300- \\
3481)\end{array}$ & $\begin{array}{l}3481- \\
3662)\end{array}$ & $\begin{array}{l}(3662- \\
3843)\end{array}$ & $\begin{array}{l}(3843- \\
4024)\end{array}$ & $\begin{array}{l}(4024- \\
4205)\end{array}$ & $\begin{array}{l}(4205- \\
4386)\end{array}$ \\
\hline $\begin{array}{c}\text { Group Two } \\
\left(\mathrm{A}_{2}\right)\end{array}$ & $\mathbf{A}_{21}$ & $\mathbf{A}_{22}$ & $\mathrm{~A}_{23}$ & $\mathbf{A}_{24}$ & $\mathbf{A}_{25}$ & $\mathbf{A}_{26}$ \\
\hline$(4386-5475))$ & $\begin{array}{l}(4386- \\
4567)\end{array}$ & $\begin{array}{l}(4567- \\
4748)\end{array}$ & $\begin{array}{l}(4748- \\
4929)\end{array}$ & $\begin{array}{l}(4929- \\
5110)\end{array}$ & $\begin{array}{l}(5110- \\
5291)\end{array}$ & $\begin{array}{l}(5291- \\
5472)\end{array}$ \\
\hline $\begin{array}{c}\text { Group } \\
\text { Three }\left(\mathrm{A}_{3}\right)\end{array}$ & $\mathbf{A}_{31}$ & $\mathbf{A}_{32}$ & $\mathbf{A}_{33}$ & $\mathbf{A}_{34}$ & $\mathbf{A}_{35}$ & $\mathbf{A}_{36}$ \\
\hline (5472-6588) & $\begin{array}{l}5472- \\
5653)\end{array}$ & $\begin{array}{l}5653- \\
5834) \\
\end{array}$ & $\begin{array}{l}(5834- \\
6015) \\
\end{array}$ & $\begin{array}{l}(6015- \\
6196)\end{array}$ & $\begin{array}{l}6196- \\
6377) \\
\end{array}$ & $\begin{array}{l}(6377- \\
6558)\end{array}$ \\
\hline $\begin{array}{c}\text { Group Four } \\
\left(\mathrm{A}_{4}\right)\end{array}$ & $\mathbf{A}_{41}$ & $\mathbf{A}_{42}$ & $\mathbf{A}_{43}$ & $\mathbf{A}_{44}$ & $\mathbf{A}_{45}$ & $\mathbf{A}_{46}$ \\
\hline (6588-7644) & $\begin{array}{l}6558- \\
6739) \\
\end{array}$ & $\begin{array}{l}(6739- \\
6920)\end{array}$ & $\begin{array}{l}(6920- \\
7101)\end{array}$ & $\begin{array}{l}(7101- \\
7282)\end{array}$ & $\begin{array}{l}(7282- \\
7463)\end{array}$ & $\begin{array}{l}(7463- \\
7644) \\
\end{array}$ \\
\hline $\begin{array}{c}\text { Group } \\
\text { Five }\left(\mathrm{A}_{5}\right)\end{array}$ & $\mathbf{A}_{51}$ & $\mathbf{A}_{52}$ & $\mathbf{A}_{53}$ & $\mathbf{A}_{54}$ & $\mathbf{A}_{55}$ & $\mathbf{A}_{56}$ \\
\hline (7644-8730) & $\begin{array}{l}(7463- \\
7825) \\
\end{array}$ & $\begin{array}{l}(7825- \\
8006) \\
\end{array}$ & $\begin{array}{l}(8006- \\
8187) \\
\end{array}$ & $\begin{array}{l}(8187- \\
8386) \\
\end{array}$ & $\begin{array}{l}(8386- \\
8549) \\
\end{array}$ & $\begin{array}{l}(8549- \\
8730) \\
\end{array}$ \\
\hline $\begin{array}{c}\text { Group } \\
\text { Six }\left(\mathrm{A}_{6}\right)\end{array}$ & $A_{61}$ & $A_{62}$ & $A_{63}$ & $A_{64}$ & $A_{65}$ & $A_{66}$ \\
\hline (8730-9816) & $\begin{array}{l}(8730- \\
8911)\end{array}$ & $\begin{array}{l}(8911- \\
9092)\end{array}$ & $\begin{array}{l}\text { (9092-- } \\
9273)\end{array}$ & $\begin{array}{l}(9273- \\
9454)\end{array}$ & $\begin{array}{l}\text { (9454- } \\
9635)\end{array}$ & $\begin{array}{l}9635- \\
9816)\end{array}$ \\
\hline
\end{tabular}

\section{Step 1}

The relative distances of the data value from each subgroup of that group have been calculated. The relative distance of the input data value is taken as the result of the subtraction of input data value from middle value of its respective subgroups as shown in step 2 of table 2 of particle swarm optimization. The relative distance value for the other elements from other subgroups has been calculated and pheromone trials stored the error of the individual data with respect to middle value of the subgroup of data group.

As for example, the minimum distance of data element (3331.597) of serial number 1 of the of table 2 is 58.903 (minimum distance of serial number one of group one is denoted by $r_{i j}(t)$ ). The middle value of that subgroup is considered as its initial estimated value and initial estimated error has to be calculated with the actual value and result is stored in the pheromone trials.

Table 2

Distance of each Element from Respective Group

\begin{tabular}{|c|c|c|c|c|c|c|c|}
\hline \multirow[b]{2}{*}{$\begin{array}{c}\text { Serial } \\
\text { No. }\end{array}$} & \multirow{2}{*}{$\begin{array}{c}\begin{array}{c}\text { Available } \\
\text { Data }\end{array} \\
\begin{array}{c}\text { Group } \\
\text { One }\left(\mathbf{A}_{1}\right)\end{array}\end{array}$} & \multicolumn{6}{|c|}{ Range of Data } \\
\hline & & $\begin{array}{c}\mathbf{A}_{11}(3300- \\
3481)\end{array}$ & $\begin{array}{c}\begin{array}{c}\mathbf{A}_{12} \\
(3481- \\
3662)\end{array} \\
\end{array}$ & $\begin{array}{c}\mathbf{A}_{13} \\
(3662- \\
3843)\end{array}$ & $\begin{array}{c}\mathbf{A}_{14} \\
(3843- \\
4024)\end{array}$ & $\begin{array}{c}\mathbf{A}_{15} \\
(4024- \\
4205)\end{array}$ & $\begin{array}{c}\mathbf{A}_{\mathbf{1 6}} \\
(4205- \\
4386)\end{array}$ \\
\hline 1 & 3331.597 & 58.903 & 239.903 & 420.903 & 601.903 & 782.903 & 963.903 \\
\hline 2 & 3734.387 & 343.887 & 162.887 & 18.113 & 199.113 & 380.113 & 561.113 \\
\hline 3 & 3912.366 & 521.866 & 340.866 & 159.866 & 21.134 & 202.134 & 383.134 \\
\hline 4 & 3939.355 & 548.855 & 367.855 & 186.855 & 5.855 & 175.145 & 356.145 \\
\hline$\frac{7}{5}$ & 3941.134 & $\frac{56.03}{550.634}$ & 369.634 & 188.634 & 7.634 & 173.366 & 354.366 \\
\hline 6 & 4009.457 & 618.957 & 437.957 & 256.957 & 75.957 & 105.043 & 286.043 \\
\hline 7 & 4066.994 & 676.494 & 495.494 & 314.494 & 133.494 & 47.506 & 228.506 \\
\hline 8 & 4079.558 & 689.058 & 508.058 & 327.058 & 146.058 & 34.942 & 215.942 \\
\hline
\end{tabular}

\begin{tabular}{|c|c|}
\hline $\begin{array}{l}9 \\
10\end{array}$ & \begin{tabular}{|l|}
4104.769 \\
4104.769
\end{tabular} \\
\hline 11 & 4104.769 \\
\hline & \\
\hline 13 & 4135.317 \\
\hline 14 & 4146.102 \\
\hline & 4174.871 \\
\hline 16 & $\begin{array}{l}4203.639 \\
\end{array}$ \\
\hline & $\begin{array}{r}4243.193 \\
4330\end{array}$ \\
\hline 19 & $\begin{array}{l}4.300 .13 \\
4318.713\end{array}$ \\
\hline 20 & 4340.285 \\
\hline & \\
\hline 21 & 4397.822 \\
\hline & \\
\hline 23 & 4466.144 \\
\hline 25 & $\begin{array}{r}4466.144 \\
446.923 \\
\end{array}$ \\
\hline 26 & $\begin{array}{l}4407.923 \\
4494.913\end{array}$ \\
\hline 27 & 4496.692 \\
\hline 28 & 45344.467 \\
\hline 29 & 4535.236 \\
\hline 31 & $\begin{array}{l}4550.014 \\
\end{array}$ \\
\hline 32 & $\frac{4352.047}{46027}$ \\
\hline 33 & 4606.347 \\
\hline 34 & $\begin{array}{l}4674.67 \\
\end{array}$ \\
\hline 35 & 4716.003 \\
\hline 36 & 4739.435 \\
\hline 37 & 4757.418 \\
\hline & 47 \\
\hline 39 & 4843.723 \\
\hline 40 & 4897.621 \\
\hline 41 & 4911.964 \\
\hline$\frac{42}{43}$ & 4922.832 \\
\hline 44 & 495.5158 \\
\hline 45 & 5034.13 \\
\hline 46 & 5061.256 \\
\hline & \\
\hline 48 & 5174.551 \\
\hline & 5188.762 \\
\hline 50 & 5228.449 \\
\hline 51 & 5248.078 \\
\hline 52 & 5268.003 \\
\hline 53 & 5285.853 \\
\hline & \\
\hline 54 & 5559.144 \\
\hline 55 & \begin{tabular}{|l|l|}
5569.929 \\
55725
\end{tabular} \\
\hline $\begin{array}{r}56 \\
57 \\
\end{array}$ & $\begin{array}{l}5577.259 \\
5616686\end{array}$ \\
\hline$\frac{31}{58}$ & $\begin{array}{l}5666.681 \\
5697.568\end{array}$ \\
\hline 59 & 5862.982 \\
\hline 60 & $\begin{array}{l}5877 \\
\end{array}$ \\
\hline 61 & 6071508 \\
\hline 62 & 6098. \\
\hline 63 & 6123.708 \\
\hline 64 & 6127 \\
\hline 65 & 6150. \\
\hline 66 & 6186.582 \\
\hline 67 & 626 \\
\hline 68 & 6261 \\
\hline 70 & 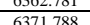 \\
\hline 71 & 6402 \\
\hline 72 & 0 \\
\hline & \\
\hline & \\
\hline 73 & 6596.518 \\
\hline & 6603.664 \\
\hline 15 & 6023 \\
\hline 70 & . \\
\hline 78 & 6066 \\
\hline 79 & \\
\hline & \\
\hline 81 & \\
\hline & 6826 \\
\hline 83 & 6833. \\
\hline 84 & 6869 \\
\hline 85 & 690 \\
\hline 86 & 691 \\
\hline & \\
\hline$\frac{60}{89}$ & \\
\hline 90 & 70 \\
\hline 91 & 70 \\
\hline 92 & 70 \\
\hline 93 & \\
\hline 94 & 71753 \\
\hline 95 & 7227. \\
\hline 96 & 7231. \\
\hline 9 & 7232 \\
\hline & 7245 \\
\hline & 7254 \\
\hline 100 & 12399 \\
\hline 102 & 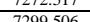 \\
\hline 410 & $\frac{12}{73}$ \\
\hline & \\
\hline & \\
\hline 106 & 7437 \\
\hline & 7454 \\
\hline 108 & 7468 \\
\hline 10 & 7497. \\
\hline 110 & 7520 \\
\hline 111 & 75 \\
\hline
\end{tabular}




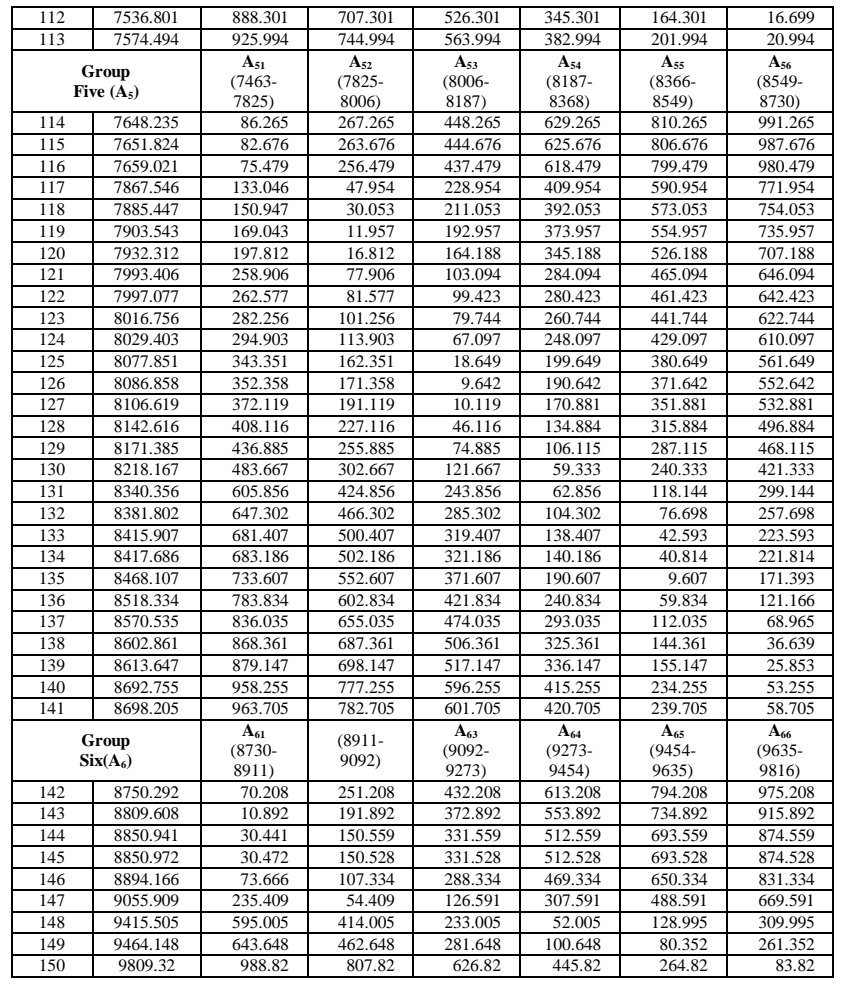

This method has been applied to every data set for calculating distance value of the all groups. Based on the minimum distance value, the group has been selected and the error with respect to that group has been calculated and stored in memory of pheromone trials and have been furnished in table 3 (column 4 and 10 respectively)

\section{Step 2}

If the error of pheromone trials is the less than $2 \%$ then middle value of the subgroup has to be considered as estimated value of data otherwise if the estimated error is not less than $2 \%$ and positive then error as calculated has to be subtracted from the earlier estimated value. If the estimated error is not less than $2 \%$ and negative then error as calculated has to be added with estimated value. The Global parameter is furnished below.

$$
r_{i j(t+1)=(1-\rho) \times} r_{i j}(t)+\rho \times \Delta r_{i j}^{g b}(t)
$$

Here $r_{i j}(t)$ is minimum distance of the particle in subgroup and $\quad \Delta r_{i j}^{g b}(t)=\frac{1}{\text { Gloabal minimum distance of the data }}$ is the global distance of the entire particle in that group.

As for an example, as the estimated error of data element (3331.597) of serial number 1 of the group one of table 3 (third column) is $1.77 \%$ (positive), the value of $r_{i j}(t+1)$ will be

$$
r_{i j^{(t+1)}}=(1-0.5) \times 58.903+0.5(1 / 5.23)=29.5471
$$

Here 3331.597 belongs to serial number one and lies in group one which contains the minimum distance value as 5.23 in the group one (according to table 2 serial no. 18 of column $\mathrm{A}_{16}$ ). Therefore the value of $\Delta r_{i j}^{g b}(t)$ is taken as 1/5.23. The estimated value has been calculated as (available data - the value of $\left.r_{i j^{(t+1)}}\right)$. Therefore the estimated value $=3331.597$ $29.5471=3302.05$ for serial no 1 . The value of $\rho$ is taken as 0.5 . The estimated error is $-0.89 \%$. This step has to be repeated until the estimated error is less than $2 \%$ or number of iteration has been reached as 50 whichever is less.

The instructions as narrated in step 2 has been repeated for other data elements of other groups to calculate the estimated value based on ant colony optimization algorithm. The error analysis has been made to produce estimated error as furnished in table 3 ( 6 and 12 column). The average error has been found as $0.73 \%$.

\begin{tabular}{|c|c|c|c|c|c|c|c|c|c|c|c|}
\hline $\begin{array}{l}\text { Sl. } \\
\text { No }\end{array}$ & $\begin{array}{c}\text { Available } \\
\text { Data }\end{array}$ & $\begin{array}{c}\text { Estimated } \\
\text { Data(initial) }\end{array}$ & $\begin{array}{c}\text { Estimated } \\
\text { Error } \\
\text { (initial) } \\
(\%) \\
\end{array}$ & $\begin{array}{l}\text { Estimated } \\
\text { data(Final) }\end{array}$ & $\begin{array}{l}\text { Estimated } \\
\text { Error }(\%)\end{array}$ & $\begin{array}{l}\text { Sl. } \\
\text { No. }\end{array}$ & $\begin{array}{l}\text { Available } \\
\text { Data }\end{array}$ & $\begin{array}{l}\text { Estimated } \\
\text { Data } \\
\text { (initial) }\end{array}$ & $\begin{array}{c}\text { Estimated } \\
\text { Error } \\
\text { (initial) }(\%)\end{array}$ & $\begin{array}{l}\text { Estimated } \\
\text { data(Final) }\end{array}$ & $\begin{array}{c}\text { Estimated } \\
\text { Error } \\
(\%)\end{array}$ \\
\hline 1 & 3331.597 & 3390.5 & 1.77 & 3302.05 & -0.89 & 76 & 6625.286 & 6648.5 & 0.35 & 6648.5 & 0.35 \\
\hline 2 & 3734.387 & 3752.5 & 0.49 & 3752.5 & 0.49 & 77 & 6664.84 & 6648.5 & -0.25 & 6648.5 & -0.25 \\
\hline 3 & 3912.366 & 3933.5 & 0.54 & 3933.5 & 0.54 & 78 & 6664.84 & 6648.5 & -0.25 & 6648.5 & -0.25 \\
\hline 4 & 3939.355 & 3933.5 & -0.15 & 3933.5 & -0.15 & 79 & 6693.527 & 6648.5 & -0.67 & 6648.5 & -0.67 \\
\hline 5 & 3941.134 & 3933.5 & -0.19 & 3933.5 & -0.19 & 80 & 6704.395 & 6648.5 & -0.83 & 6648.5 & -0.83 \\
\hline 6 & 4009.457 & 3933.5 & -1.89 & 3933.5 & -1.89 & 81 & 6770.939 & 6829.5 & 0.86 & 6829.5 & 0.86 \\
\hline 7 & 4066.994 & 4114.5 & 1.17 & 4114.5 & 1.17 & 82 & 6826.615 & 6829.5 & 0.04 & 6829.5 & 0.04 \\
\hline 8 & 4079.558 & 4114.5 & 0.86 & 4114.5 & 0.86 & 83 & 6833.812 & 6829.5 & -0.06 & 6829.5 & -0.06 \\
\hline 9 & 4104.769 & 4114.5 & 0.24 & 4114.5 & 0.24 & 84 & 6869.809 & 6829.5 & -0.59 & 6829.5 & -0.59 \\
\hline 10 & 4104.769 & 4114.5 & 0.24 & 4114.5 & 0.24 & 85 & 6900.356 & 6829.5 & -1.03 & 6829.5 & -1.03 \\
\hline 11 & 4104.769 & 4114.5 & 0.24 & 4114.5 & 0.24 & 86 & 6912.92 & 6829.5 & -1.21 & 6829.5 & -1.21 \\
\hline 12 & 4135.317 & 4114.5 & -0.50 & 4114.5 & -0.50 & 87 & 6941.689 & 7010.5 & 0.99 & 7010.5 & 0.99 \\
\hline 13 & 4135.317 & 4114.5 & -0.50 & 4114.5 & -0.50 & 88 & 6979.464 & 7010.5 & 0.44 & 7010.5 & 0.44 \\
\hline 14 & 4146.102 & 4114.5 & -0.76 & 4114.5 & -0.76 & 89 & 6997.447 & 7010.5 & 0.19 & 7010.5 & 0.19 \\
\hline 15 & 4174.871 & 4114.5 & -1.45 & 4114.5 & -1.45 & 90 & 7008.233 & 7010.5 & 0.03 & 7010.5 & 0.03 \\
\hline 16 & 4203.639 & 4114.5 & -2.12 & 4143.55 & -1.43 & 91 & 7037.001 & 7010.5 & -0.38 & 7010.5 & -0.38 \\
\hline 17 & 4243.193 & 4295.5 & 1.23 & 4295.5 & 1.23 & 92 & 7038.78 & 7010.5 & -0.40 & 7010.5 & -0.40 \\
\hline 18 & 4300.73 & 4295.5 & -0.12 & 4295.5 & -0.12 & 93 & 7038.78 & 7010.5 & -0.40 & 7010.5 & -0.40 \\
\hline 19 & 4318.713 & 4295.5 & -0.54 & 4295.5 & -0.54 & 94 & 7175.344 & 7191.5 & 0.23 & 7191.5 & 0.23 \\
\hline 20 & 4340.285 & 4295.5 & -1.03 & 4295.5 & -1.03 & 95 & 7227.544 & 7191.5 & -0.50 & 7191.5 & -0.50 \\
\hline 21 & 4397.822 & 4476.5 & 1.79 & 4476.5 & 1.79 & 96 & 7231.102 & 7191.5 & -0.55 & 7191.5 & -0.55 \\
\hline 22 & 4426.59 & 4476.5 & 1.13 & 4476.5 & 1.13 & 97 & 7232.962 & 7191.5 & -0.57 & 7191.5 & -0.57 \\
\hline 23 & 4466.144 & 4476.5 & 0.23 & 4476.5 & 0.23 & 98 & 7245.445 & 7191.5 & -0.74 & 7191.5 & -0.74 \\
\hline 24 & 4466.144 & 4476.5 & 0.23 & 4476.5 & 0.23 & 99 & 7254.534 & 7191.5 & -0.87 & 7191.5 & -0.87 \\
\hline 25 & 4467.923 & 4476.5 & 0.19 & 4476.5 & 0.19 & 100 & 7259.87 & 7191.5 & -0.94 & 7191.5 & -0.94 \\
\hline
\end{tabular}

Table 3. Estimated Output and Estimated Error Based on ACO 


\begin{tabular}{|c|c|c|c|c|c|c|c|c|c|c|c|}
\hline 26 & 4494.913 & 4476.5 & -0.41 & 4476.5 & -0.41 & 101 & 7272.517 & 7191.5 & -1.11 & 7191.5 & -1.11 \\
\hline 27 & 4496.692 & 4476.5 & -0.45 & 4476.5 & -0.45 & 102 & 7299.506 & 7372.5 & 1.00 & 7372.5 & 1.00 \\
\hline 28 & 4534.467 & 4476.5 & -1.28 & 4476.5 & -1.28 & 103 & 7331.863 & 7372.5 & 0.55 & 7372.5 & 0.55 \\
\hline 29 & 4563.236 & 4476.5 & -1.90 & 4476.5 & -1.90 & 104 & 7409.162 & 7372.5 & -0.49 & 7372.5 & -0.49 \\
\hline 30 & 4565.014 & 4476.5 & -1.94 & 4476.5 & -1.94 & 105 & 7437.849 & 7372.5 & -0.88 & 7372.5 & -0.88 \\
\hline 31 & 4592.004 & 4657.5 & 1.43 & 4657.5 & 1.43 & 106 & 7437.849 & 7372.5 & $\begin{array}{l}-0.88 \\
\end{array}$ & 7372.5 & -0.88 \\
\hline 32 & 4602.79 & 4657.5 & 1.19 & 4657.5 & 1.19 & 107 & 7454.053 & 7372.5 & -1.09 & 7372.5 & -1.09 \\
\hline 33 & 4606.347 & 4657.5 & 1.11 & 4657.5 & 1.11 & 108 & 7468.396 & 7553.5 & 1.14 & 7553.5 & 1.14 \\
\hline 34 & 4674.67 & 4657.5 & -0.37 & 4657.5 & -0.37 & 109 & 7497.246 & 7553.5 & 0.75 & 7553.5 & 0.75 \\
\hline 35 & 4716.003 & 4657.5 & -1.24 & 4657.5 & -1.24 & 110 & 7520.597 & 7553.5 & 0.44 & 7553.5 & 0.44 \\
\hline 36 & 4739.435 & 4657.5 & -1.73 & 4657.5 & -1.73 & 111 & 7522.375 & 7553.5 & 0.41 & 7553.5 & 0.41 \\
\hline 37 & 4757.418 & 4838.5 & 1.70 & 4838.5 & 1.70 & 112 & 7536.801 & 7553.5 & 0.22 & 7553.5 & 0.22 \\
\hline 38 & 4757.418 & 4838.5 & 1.70 & 4838.5 & 1.70 & 113 & 7574.494 & 7553.5 & -0.28 & 7553.5 & -0.28 \\
\hline 39 & 4843.723 & 4838.5 & $\begin{array}{l}-0.11 \\
\end{array}$ & 4838.5 & $\begin{array}{l}-0.11 \\
\end{array}$ & 114 & 7648.235 & 7734.5 & 1.13 & 7734.5 & 1.13 \\
\hline 40 & 4897.621 & 4838.5 & -1.21 & 4838.5 & -1.21 & 115 & 7651.824 & 7734.5 & 1.08 & 7734.5 & 1.08 \\
\hline 41 & 4911.964 & 4838.5 & -1.50 & 4838.5 & -1.50 & 116 & 7659.021 & 7734.5 & 0.99 & 7734.5 & 0.99 \\
\hline 42 & 4922.832 & 4838.5 & -1.71 & 4838.5 & -1.71 & 117 & 7867.546 & 7915.5 & 0.61 & 7915.5 & 0.61 \\
\hline 43 & 4924.611 & 4838.5 & -1.75 & 4838.5 & -1.75 & 118 & 7885.447 & 7915.5 & 0.38 & 7915.5 & 0.38 \\
\hline 44 & 4955.158 & 5019.5 & 1.30 & 5019.5 & 1.30 & 119 & $\begin{array}{l}7903.543 \\
\end{array}$ & 7915.5 & 0.15 & 7915.5 & 0.15 \\
\hline 45 & 5034.134 & 5019.5 & -0.29 & 5019.5 & -0.29 & 120 & 7932.312 & 7915.5 & -0.21 & 7915.5 & -0.21 \\
\hline 46 & 5061.256 & 5019.5 & $\begin{array}{l}-0.83 \\
\end{array}$ & 5019.5 & $\begin{array}{l}-0.83 \\
\end{array}$ & 121 & 7993.406 & 7915.5 & $\begin{array}{l}-0.97 \\
\end{array}$ & 7915.5 & -0.97 \\
\hline 47 & 5075.681 & 5019.5 & -1.11 & 5019.5 & -1.11 & 122 & 7997.077 & 7915.5 & -1.02 & 7915.5 & -1.02 \\
\hline 48 & 5174.551 & 5200.5 & 0.50 & 5200.5 & 0.50 & 123 & 8016.756 & 8096.5 & 0.99 & 8096.5 & 0.99 \\
\hline 49 & 5188.762 & 5200.5 & 0.23 & 5200.5 & 0.23 & 124 & 8029.403 & 8096.5 & 0.84 & 8096.5 & 0.84 \\
\hline 50 & 5228.449 & 5200.5 & -0.53 & 5200.5 & -0.53 & 125 & 8077.851 & 8096.5 & 0.23 & 8096.5 & 0.23 \\
\hline 51 & 5248.078 & 5200.5 & -0.91 & 5200.5 & -0.91 & 126 & 8086.858 & 8096.5 & 0.12 & 8096.5 & 0.12 \\
\hline 52 & 5268.003 & 5200.5 & -1.28 & 5200.5 & -1.28 & 127 & 8106.619 & 8096.5 & -0.12 & 8096.5 & -0.12 \\
\hline 53 & 5285.853 & 5200.5 & -1.61 & 5200.5 & -1.61 & 128 & 8142.616 & 8096.5 & -0.57 & 8096.5 & -0.57 \\
\hline 54 & 5559.144 & 5562.5 & 0.06 & 5562.5 & 0.06 & 129 & 8171.385 & 8096.5 & -0.92 & 8096.5 & -0.92 \\
\hline 55 & 5569.929 & 5562.5 & -0.13 & 5562.5 & $\begin{array}{l}-0.13 \\
\end{array}$ & 130 & 8218.167 & 8277.5 & 0.72 & 8277.5 & 0.72 \\
\hline 56 & 5577.259 & 5562.5 & -0.26 & 5562.5 & -0.26 & 131 & 8340.356 & 8277.5 & -0.75 & 8277.5 & -0.75 \\
\hline 57 & 5616.681 & 5562.5 & -0.96 & 5562.5 & -0.96 & 132 & 8381.802 & 8458.5 & 0.92 & 8458.5 & 0.92 \\
\hline 58 & 5697.568 & 5743.5 & 0.81 & 5743.5 & 0.81 & 133 & 8415.907 & 8458.5 & 0.51 & 8458.5 & 0.51 \\
\hline 59 & 5862.982 & 5924.5 & 1.05 & 5924.5 & 1.05 & 134 & 8417.686 & 8458.5 & 0.48 & 8458.5 & 0.48 \\
\hline 60 & 5877.325 & 5924.5 & 0.80 & 5924.5 & 0.80 & 135 & 8468.107 & 8458.5 & $\begin{array}{l}-0.11 \\
\end{array}$ & 8458.5 & -0.11 \\
\hline 61 & 6071.508 & 6105.5 & 0.56 & 6105.5 & 0.56 & 136 & 8518.334 & 8639.5 & 1.42 & 8639.5 & 1.42 \\
\hline 62 & 6098.497 & 6105.5 & 0.11 & 6105.5 & 0.11 & 137 & 8570.535 & 8639.5 & 0.80 & 8639.5 & 0.80 \\
\hline 63 & 6123.708 & 6105.5 & -0.30 & 6105.5 & -0.30 & 138 & 8602.861 & 8639.5 & 0.43 & 8639.5 & 0.43 \\
\hline 64 & 6127.266 & 6105.5 & -0.36 & 6105.5 & -0.36 & 139 & 8613.647 & 8639.5 & 0.30 & 8639.5 & 0.30 \\
\hline 65 & 6150.616 & 6105.5 & -0.73 & 6105.5 & -0.73 & 140 & 8692.755 & 8639.5 & -0.61 & 8639.5 & -0.61 \\
\hline 66 & 6186.582 & 6105.5 & -1.31 & 6105.5 & -1.31 & 141 & 8698.205 & 8639.5 & $\begin{array}{l}-0.67 \\
\end{array}$ & 8639.5 & -0.67 \\
\hline 67 & 6262.05 & 6286.5 & 0.39 & 6286.5 & 0.39 & 142 & 8750.292 & 8820.5 & 0.80 & 8820.5 & 0.80 \\
\hline 68 & 6267.469 & 6286.5 & 0.30 & 6286.5 & 0.30 & 143 & 8809.608 & 8820.5 & 0.12 & 8820.5 & 0.12 \\
\hline 69 & 6362.781 & 6286.5 & -1.20 & 6286.5 & -1.20 & 144 & 8850.941 & 8820.5 & -0.34 & 8820.5 & -0.34 \\
\hline 70 & 6371.788 & 6286.5 & -1.34 & 6286.5 & -1.34 & 145 & 8850.972 & 8820.5 & $\begin{array}{l}-0.34 \\
\end{array}$ & 8820.5 & -0.34 \\
\hline 71 & 6402.335 & 6467.5 & 1.02 & 6467.5 & 1.02 & 146 & 8894.166 & 8820.5 & -0.83 & 8820.5 & -0.83 \\
\hline 72 & 6431.104 & 6467.5 & 0.57 & 6467.5 & 0.57 & 147 & 9055.909 & 9001.5 & -0.60 & 9001.5 & -0.60 \\
\hline 73 & 6596.518 & 6648.5 & 0.79 & 6648.5 & 0.79 & 148 & 9415.505 & 9363.5 & -0.55 & 9363.5 & -0.55 \\
\hline 74 & 6603.664 & 6648.5 & 0.68 & 6648.5 & 0.68 & 149 & 9464.148 & 9363.5 & -1.06 & 9363.5 & -1.06 \\
\hline 75 & 6623.507 & 6648.5 & 0.38 & 6648.5 & 0.38 & 150 & 9809.32 & 9725.5 & -0.85 & 9725.5 & -0.85 \\
\hline
\end{tabular}

\subsection{Statistical Methods and Soft computing models}

The statistical method using least square technique (LSQ) based on linear, exponential, asymptotic, curvilinear and logarithmic equations have been applied on the cumulative antecedent item to produce estimated cumulative antecedent item. The error analysis has been made to compute estimated error and average error. The estimated data based on statistical methods and the estimated errors have been furnished in table 7. It has been observed that the least square technique based on logarithmic equation has given minimum error as compared to other statistical models. The problem of using this model is that if original data (actual data) is not stabilized, some noise may enter with the original data, and as a result that may generate some erroneous results. This sort of unstabilized data can be avoided by using soft computing models.
Under soft computing domain, the harmony search, fuzzy logic, neural network, particle swarm optimization, artificial bee colony algorithm have been used to produce estimated data based on that models.

\subsection{Review of models}

The statistical methods, fuzzy logic, neural network, particle swarm optimization, artificial bee colony algorithm have been used to produce estimated data based on those models. The error analysis and the parameters of residual analysis have been applied on the estimated data with respect to the available data. The error analysis includes the computation of average error and residual analysis includes the computation of sum of absolute residual, mean of absolute residual, mean of mean of absolute residual, median of absolute residual, maximum of absolute residual and standard deviation of absolute residual. The average error and the parameters of residual analysis have been furnished in table 4 . The strength of each model can be ascertained by the error and residual 
analysis. The less value of the parameter for the error and residual analysis indicates more strength of the model (method) than others. From table 4 it has been observed that out of seven cases, ACO has been preferred by seven cases. Therefore the ACO has been selected as the preferable optimizing model for the estimation of data. Therefore, the estimated data based on ACO can be used for the extraction of knowledge. The estimated data based on the ACO has been named as intermediate data and that name will be used in further processing.

Table 4

Average Error and Residual Analysis of Applied Methods

\begin{tabular}{|c|c|c|c|c|c|c|c|c|}
\hline \multicolumn{1}{|c|}{ on Iris Flowers Data } \\
\hline $\begin{array}{c}\text { Serial } \\
\text { Number }\end{array}$ & $\begin{array}{c}\text { Method } \\
\text { Name }\end{array}$ & $\begin{array}{c}\text { Sum of } \\
\text { Absolute } \\
\text { Residual }\end{array}$ & $\begin{array}{c}\text { Maximum } \\
\text { Absolute } \\
\text { Residual }\end{array}$ & $\begin{array}{c}\text { Mean } \\
\text { Absolute } \\
\text { Residual }\end{array}$ & $\begin{array}{c}\text { Mean of } \\
\text { Mean } \\
\text { Absolute } \\
\text { Residual }\end{array}$ & $\begin{array}{c}\text { Median } \\
\text { of } \\
\text { Absolute } \\
\text { Residual }\end{array}$ & $\begin{array}{c}\text { Standard } \\
\text { Deviation } \\
\text { of } \\
\text { Absolute } \\
\text { residual }\end{array}$ & $\begin{array}{c}\text { Average } \\
\text { Error (\%) }\end{array}$ \\
\hline 1 & $\begin{array}{c}\text { Linear } \\
\text { Equation }\end{array}$ & 20090 & 710.349 & 3.375 & 0.023 & 286.376 & 94.90468 & 2.25 \\
\hline 2 & $\begin{array}{c}\text { Exponential } \\
\text { equation }\end{array}$ & 31627.33 & 618.573 & 4.907 & 0.033 & 495.577 & 144.1412 & 3.27 \\
\hline 3 & $\begin{array}{c}\text { Asymptotic } \\
\text { Equation }\end{array}$ & 80269.16 & 2191.526 & 15.404 & 0.103 & 309.903 & 459.805 & 10.27 \\
\hline 4 & $\begin{array}{c}\text { Curvilinear } \\
\text { Equation }\end{array}$ & 20066.95 & 716.8084 & 3.382 & 0.023 & 282.7374 & 95.0663 & 2.25 \\
\hline 5 & $\begin{array}{c}\text { Logarithm } \\
\text { Equation }\end{array}$ & 27677.26 & 998.64 & 4.762 & 0.032 & 71.827 & 143.9862 & 3.17 \\
\hline 6 & $\begin{array}{c}\text { Fuzzy } \\
\text { Logic }\end{array}$ & 14493.82 & 420.903 & 2.429 & 0.016 & 65.507 & 64.14068 & 1.62 \\
\hline 7 & $\begin{array}{c}\text { Neural } \\
\text { Network }\end{array}$ & 6811.231 & 191.861 & 1.104 & 0.007 & 24.993 & 28.12644 & 0.78 \\
\hline 8 & PSO & 8917.629 & 324.073 & 1.529 & 0.01 & 63.043 & 53.0546 & 1.02 \\
\hline 9 & ACO & $\underline{6728.986}$ & $\underline{121.166}$ & $\underline{1.098}$ & $\underline{0.007}$ & $\underline{24.993}$ & $\underline{26.82716}$ & $\underline{0.73}$ \\
\hline
\end{tabular}

\subsection{Creation of Knowledge}

Certain unknown data have been taken for the extraction of knowledge for that data item and that have been furnished in table 5 . The preprocessing technique namely decimal scaling techniques have been applied on unknown data sets to convert it into proper format for the extraction of knowledge. The unknown data have been termed as tested data. The purpose of this work is to assess the quality of flowers (unknown data) based on the information A, B, C. The proposed work is to estimate the value $\mathrm{D}$ based on these primary values i.e. A, B, $\mathrm{C}$ using the proposed model. Thereafter an effort has to be made the quality of information which has been obtained (estimated value of $\mathrm{D}$ ) by calculating the error of the estimated value of $\mathrm{D}$ with respect to actual value of $\mathrm{D}$. The items sepal width, petal length petal width and sepal width have been termed as A, B, C and D respectively.

Table 5

Unknown Data

\begin{tabular}{|r|r|r|r|r|r|r|r|r|r|}
\hline $\begin{array}{c}\text { Serial } \\
\text { Number }\end{array}$ & A & B & \multicolumn{1}{c|}{ C } & D & $\begin{array}{c}\text { Serial } \\
\text { Number }\end{array}$ & A & B & C & D \\
\hline 1 & 2.65 & 1.2 & 0.2 & 4.4 & 31 & 3 & 4.55 & 1.45 & 5.85 \\
\hline 2 & 2.95 & 1.25 & 0.15 & 4.35 & 32 & 2.6 & 5.05 & 1.75 & 6.15 \\
\hline 3 & 3.45 & 1.55 & 0.2 & 5 & 33 & 2.7 & 5 & 1.7 & 6.15 \\
\hline 4 & 3.4 & 1.6 & 0.3 & 5.4 & 34 & 2.95 & 4.75 & 1.65 & 6.45 \\
\hline 5 & 3.35 & 1.65 & 0.45 & 5.05 & 35 & 3.15 & 4.7 & 1.45 & 6.85 \\
\hline 6 & 3.35 & 1.8 & 0.35 & 4.95 & 36 & 2.9 & 5.15 & 1.4 & 6.55 \\
\hline 7 & 1.9 & 3.35 & 1.1 & 3.85 & 37 & 2.85 & 5.25 & 1.45 & 6.5 \\
\hline 8 & 3.85 & 1.4 & 0.35 & 5.25 & 38 & 3.1 & 4.7 & 1.8 & 5.8 \\
\hline 9 & 2.95 & 2.4 & 0.7 & 5.2 & 39 & 3.2 & 4.75 & 1.65 & 6.35 \\
\hline 10 & 4.15 & 1.45 & 0.15 & 5.35 & 40 & 3 & 5 & 1.75 & 6.35 \\
\hline 11 & 3.25 & 2.35 & 0.6 & 5.25 & 41 & 2.95 & 5.05 & 1.85 & 6.15 \\
\hline 12 & 3.15 & 2.45 & 0.75 & 5.1 & 42 & 2.95 & 5.55 & 1.8 & 6.4 \\
\hline 13 & 3.1 & 2.6 & 0.7 & 5 & 43 & 3.1 & 5.3 & 1.9 & 6.5 \\
\hline 14 & 2.4 & 3.5 & 1 & 5.2 & 44 & 3.1 & 5.45 & 1.8 & 6.85 \\
\hline 15 & 2.3 & 3.85 & 1 & 5.75 & 45 & 2.9 & 5.7 & 1.85 & 6.8 \\
\hline 16 & 3.3 & 2.75 & 0.7 & 5.85 & 46 & 2.95 & 5.55 & 1.95 & 6.4 \\
\hline 17 & 3.5 & 2.5 & 0.7 & 5.7 & 47 & 3.05 & 5.35 & 2.05 & 6.55 \\
\hline 18 & 2.5 & 3.65 & 1.05 & 5.6 & 48 & 2.9 & 5.4 & 2.25 & 6.55 \\
\hline 19 & 2.7 & 4.2 & 1.25 & 5.8 & 49 & 2.95 & 5.35 & 2.25 & 6.65 \\
\hline 20 & 2.65 & 4.3 & 1.25 & 5.55 & 50 & 3 & 5.7 & 2.1 & 6.9 \\
\hline 21 & 2.55 & 4.6 & 1.4 & 5.85 & 51 & 3.05 & 5.8 & 2.05 & 6.85 \\
\hline
\end{tabular}

\begin{tabular}{|r|r|r|r|r|r|r|r|r|r|}
\hline 22 & 2.6 & 4.6 & 1.35 & 5.85 & 52 & 2.95 & 6.1 & 1.95 & 7.2 \\
\hline 23 & 3 & 4.15 & 1.25 & 5.65 & 53 & 3 & 5.85 & 2.15 & 6.8 \\
\hline 24 & 2.95 & 4.2 & 1.3 & 6 & 54 & 3.35 & 5.55 & 2.2 & 6.45 \\
\hline 25 & 2.9 & 4.3 & 1.3 & 6.3 & 55 & 3.3 & 5.55 & 2.3 & 6.55 \\
\hline 26 & 2.7 & 4.4 & 1.5 & 5.55 & 56 & 3.1 & 5.9 & 2.3 & 7.3 \\
\hline 27 & 2.65 & 4.5 & 1.5 & 5.3 & 57 & 3.15 & 6.3 & 2.3 & 6.95 \\
\hline 28 & 2.75 & 4.55 & 1.5 & 6.1 & 58 & 3.45 & 6.05 & 2.5 & 6.75 \\
\hline 29 & 2.95 & 4.4 & 1.4 & 6.25 & 59 & 3.7 & 6.25 & 2.25 & 7.55 \\
\hline 30 & 2.95 & 4.65 & 1.4 & 6.1 & 60 & 3.8 & 6.55 & 2.1 & 7.8 \\
\hline
\end{tabular}

Step 2

Now, it is necessary to calculate cumulative antecedent item using the relation as formed in section of factor analysis. The relation has been furnished as

Total effect value $=(0.970912) \times \mathrm{A}+(0.683227) \times \mathrm{B}+$ $(0.701015) \times$ C .

Step 3

Now using the relation, the total effect value has been computed and furnished in table 6 .

Table 6

Total Effect Value

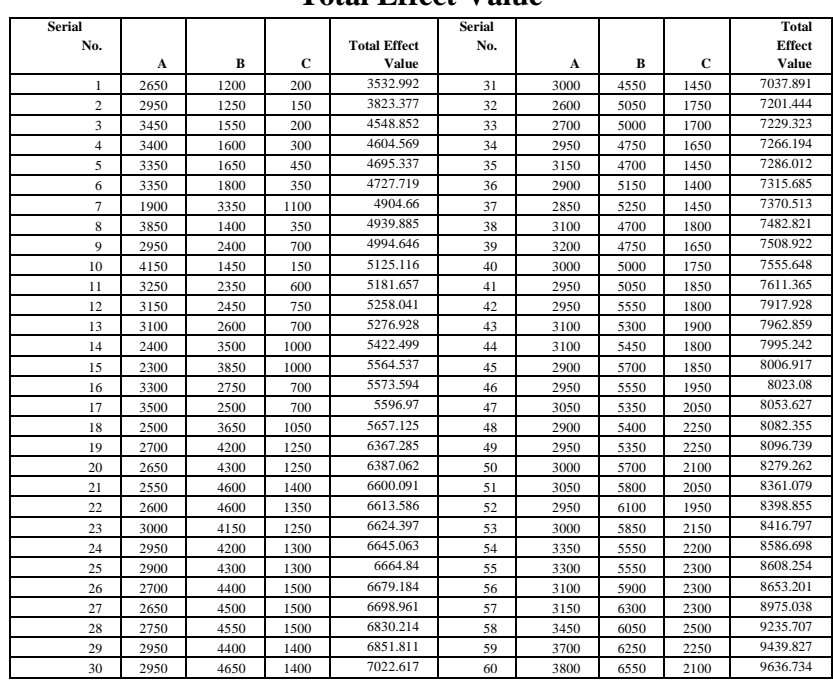

\section{Step 4}

The cumulative antecedent item for tested data has been furnished in table 6 which has to be processed in subsequent sections. The cumulative antecedent item has been termed as tested antecedent data. The tested antecedent data has been furnished in table 7 .

Table 7

Tested Data

\begin{tabular}{|l|c|c|c|c|c|c|c|}
\hline $\begin{array}{l}\text { Serial } \\
\text { Numbe } \\
\mathbf{r}\end{array}$ & $\begin{array}{l}\text { Tested } \\
\text { Antecede } \\
\text { nt } \\
\text { Data }\end{array}$ & $\begin{array}{l}\text { Serial } \\
\text { Numbe } \\
\mathbf{r}\end{array}$ & $\begin{array}{l}\text { Tested } \\
\text { Antecede } \\
\text { nt } \\
\text { Data }\end{array}$ & $\begin{array}{l}\text { Serial } \\
\text { Numbe } \\
\mathbf{r}\end{array}$ & $\begin{array}{l}\text { Tested } \\
\text { Antecede } \\
\text { nt } \\
\text { Data }\end{array}$ & $\begin{array}{l}\text { Serial } \\
\text { Numbe } \\
\mathbf{r}\end{array}$ & $\begin{array}{l}\text { Tested } \\
\text { Antecede } \\
\text { nt } \\
\text { Data }\end{array}$ \\
\hline 1 & 3532.992 & 16 & 5573.594 & 31 & 7037.891 & 46 & 8023.08 \\
\hline 2 & 3823.377 & 17 & 5596.97 & 32 & 7201.444 & 47 & 8053.627 \\
\hline 3 & 4548.852 & 18 & 5657.125 & 33 & 7229.323 & 48 & 8082.355 \\
\hline 4 & 4604.569 & 19 & 6367.285 & 34 & 7266.194 & 49 & 8096.739 \\
\hline 5 & 4695.337 & 20 & 6387.062 & 35 & 7286.012 & 50 & 8279.262 \\
\hline 6 & 4727.719 & 21 & 6600.091 & 36 & 7315.685 & 51 & 8361.079 \\
\hline 7 & 4904.66 & 22 & 6613.586 & 37 & 7370.513 & 52 & 8398.855 \\
\hline 8 & 4939.885 & 23 & 6624.397 & 38 & 7482.821 & 53 & 8416.797 \\
\hline 9 & 4994.646 & 24 & 6645.063 & 39 & 7508.922 & 54 & 8586.698 \\
\hline 10 & 5125.116 & 25 & 6664.84 & 40 & 7555.648 & 55 & 8608.254 \\
\hline 11 & 5181.657 & 26 & 6679.184 & 41 & 761.365 & 56 & 8653.201 \\
\hline 12 & 5258.041 & 27 & 6698.961 & 42 & 7917.928 & 57 & 8975.038 \\
\hline 13 & 5276.928 & 28 & 6830.214 & 43 & 7962.859 & 58 & 9235.707 \\
\hline 14 & 5422.499 & 29 & 6851.811 & 44 & 7995.242 & 59 & 9439.827 \\
\hline 15 & 5564.537 & 30 & 7022.617 & 45 & 8006.917 & 60 & 9636.734 \\
\hline
\end{tabular}

Step 5

The statistical methods and soft computing models have been applied on the tested antecedent data. The statistical methods include least square technique (LSQ) based on linear, exponential, asymptotic, curvilinear and logarithmic 
equations. The soft computing models include fuzzy logic (Fuzzy), neural network (NN), genetic algorithm(GA), particle swarm optimization (PSO), ant colony optimization(ACO. The average errors based on statistical and soft computing models have been furnished in tables 8 .

Table 8

Average Error and Residual Analysis using Tested Data (Iris Flowers Data)

\begin{tabular}{|c|c|c|c|r|r|r|r|r|}
\hline $\begin{array}{c}\text { Serial } \\
\text { Number }\end{array}$ & $\begin{array}{c}\text { Method } \\
\text { Name }\end{array}$ & $\begin{array}{c}\text { Sum of } \\
\text { Absolute } \\
\text { Residual }\end{array}$ & $\begin{array}{c}\text { Maximum } \\
\text { Absolute } \\
\text { Residual }\end{array}$ & $\begin{array}{c}\text { Mean } \\
\text { Absolute } \\
\text { Residual }\end{array}$ & $\begin{array}{c}\text { Mean of } \\
\text { Mean } \\
\text { Absolute } \\
\text { Residual }\end{array}$ & $\begin{array}{c}\text { Median } \\
\text { of } \\
\text { obsolute } \\
\text { Residual }\end{array}$ & $\begin{array}{c}\text { Standard } \\
\text { Deviation } \\
\text { of } \\
\text { Absolute } \\
\text { residual }\end{array}$ & $\begin{array}{c}\text { Average } \\
\text { Error } \\
(\%)\end{array}$ \\
\hline 1 & $\begin{array}{c}\text { Linear } \\
\text { Equation }\end{array}$ & 8978.615 & 555.933 & 1.41 & 0.02 & 85.876 & 100.0384 & 2.34 \\
\hline 2 & $\begin{array}{c}\text { Exponential } \\
\text { equation }\end{array}$ & 13170.25 & 526.431 & 1.97 & 0.03 & 264.337 & 140.3142 & 3.29 \\
\hline 3 & $\begin{array}{c}\text { Asymptotic } \\
\text { Equation }\end{array}$ & 26623.52 & 2161.037 & 4.46 & 0.07 & 517.588 & 346.7657 & 7.43 \\
\hline 4 & $\begin{array}{c}\text { Curvilinear } \\
\text { Equation }\end{array}$ & 8970.36 & 562.254 & 1.41 & 0.02 & 82.555 & 100.4892 & 2.34 \\
\hline 5 & $\begin{array}{c}\text { Logarithm } \\
\text { Equation }\end{array}$ & 11435.6 & 835.499 & 1.74 & 0.03 & 162.808 & 155.382 & 2.9 \\
\hline 6 & $\begin{array}{c}\text { Fuzzy } \\
\text { Logic }\end{array}$ & 411009.6 & 9636.734 & 60 & 1 & 7022.617 & 1470.556 & 1.43 \\
\hline 7 & $\begin{array}{c}\text { Neural } \\
\text { Network }\end{array}$ & 2928.87 & 180.785 & 0.63 & 0.01 & 12.317 & 28.8091 & 0.59 \\
\hline 8 & PSO & 3380.681 & 253.008 & 0.54 & 0.01 & $\underline{4.237}$ & 50.62961 & 0.91 \\
\hline 9 & ACO & $\underline{2081.524}$ & $\underline{92.234}$ & $\underline{0.32}$ & $\underline{0.01}$ & $\underline{12.117}$ & $\underline{26.11567}$ & $\underline{0.53}$ \\
\hline
\end{tabular}

\subsection{Extraction of Knowledge}

The estimated ACO output of the tested data has been furnished in table 9. The data (serial number 1 of the table 9) is 3571.5 which lie between (serial number 1 and 2 of table 6). Therefore its estimated output of the consequent item will be the average of actual output of the consequent item of table 6 (serial number 1 and 2). Similarly output (estimated consequent item) of the all the tested data has been calculated and furnished in table 12 .

Table 9

Tested with ACO output on tested data

\begin{tabular}{|c|r|r|r|r|r|r|r|}
\hline $\begin{array}{l}\text { Tested } \\
\text { Antecedent } \\
\text { Data }\end{array}$ & ACO & $\begin{array}{l}\text { Tested } \\
\text { Antecedent } \\
\text { Data }\end{array}$ & ACO & $\begin{array}{l}\text { Tested } \\
\text { Antecedent } \\
\text { Data }\end{array}$ & ACO & \multicolumn{1}{|c|}{$\begin{array}{l}\text { Tested } \\
\text { Antecedent } \\
\text { Data }\end{array}$} & ACO \\
\hline 3532.992 & 3571.5 & 5573.594 & 5562.5 & 7037.891 & 7191.5 & 8023.08 & 8096.5 \\
\hline 3823.377 & 3843 & 5596.97 & 5653 & 7201.444 & 7191.5 & 8053.627 & 8096.5 \\
\hline 4548.852 & 4476.5 & 5657.125 & 6286.5 & 7229.323 & 7191.5 & 8082.355 & 8096.5 \\
\hline 4604.569 & 4657.5 & 6367.285 & 6377 & 7266.194 & 7282 & 8096.739 & 8277.5 \\
\hline 4695.337 & 4657.5 & 6387.062 & 6648.5 & 7286.012 & 7372.5 & 8279.262 & 8368 \\
\hline 4727.719 & 4862.75 & 6600.091 & 6648.5 & 7315.685 & 7372.5 & 8361.079 & 8458.5 \\
\hline 4904.66 & 5019.5 & 6613.586 & 6648.5 & 7370.513 & 7553.5 & 8398.855 & 8458.5 \\
\hline 4939.885 & 5110 & 6624.397 & 6648.5 & 7482.821 & 7553.5 & 8416.797 & 8639.5 \\
\hline 4994.646 & 5200.5 & 6645.063 & 6648.5 & 7508.922 & 7553.5 & 8586.698 & 8639.5 \\
\hline 5125.116 & 5200.5 & 6664.84 & 6648.5 & 7555.648 & 7644 & 8608.254 & 8639.5 \\
\hline 5181.657 & 5200.5 & 6679.184 & 6648.5 & 7611.365 & 7915.5 & 8653.201 & 8911 \\
\hline 5258.041 & 5381.5 & 6698.961 & 6829.5 & 7917.928 & 7915.5 & 8975.038 & 9182.5 \\
\hline 5276.928 & 5562.5 & 6830.214 & 6829.5 & 7962.859 & 7915.5 & 9235.707 & 9363.5 \\
\hline 5422.499 & 5562.5 & 6851.811 & 7010.5 & 7995.242 & 8006 & 9439.827 & 9544.5 \\
\hline 5564.537 & 5569.5 & 7022.617 & 7010.5 & 8006.917 & 8096.5 & 9636.734 & 9594.5 \\
\hline
\end{tabular}

Table 10

Estimated Error on Tested Data by ACO

\begin{tabular}{|c|c|r|r|c|c|}
\hline $\begin{array}{c}\text { Serial } \\
\text { Number }\end{array}$ & $\begin{array}{c}\text { Tested } \\
\text { Data }\end{array}$ & $\begin{array}{c}\text { Tested data } \\
\text { (ACO) }\end{array}$ & $\begin{array}{c}\text { Actual } \\
\text { Consequent } \\
\text { Item }\end{array}$ & $\begin{array}{c}\text { Estimated } \\
\text { Consequent } \\
\text { Item }\end{array}$ & $\begin{array}{c}\text { Estimated } \\
\text { Error (\%) }\end{array}$ \\
\hline 1 & 3532.992 & 3571.5 & 5573.594 & 4500 & 2.27 \\
\hline 2 & 3823.377 & 3843 & 5596.97 & 4300 & -1.15 \\
\hline 3 & 4548.852 & 4476.5 & 5657.125 & 5150 & 3.00 \\
\hline 4 & 4604.569 & 4657.5 & 6367.285 & 5150 & -4.63 \\
\hline 5 & 4695.337 & 4657.5 & 6387.062 & 4966.67 & -1.65 \\
\hline 6 & 4727.719 & 4862.75 & 6600.091 & 4966.67 & 0.34 \\
\hline 7 & 4904.66 & 5019.5 & 6613.586 & 5150 & 33.77 \\
\hline 8 & 4939.885 & 5110 & 6624.397 & 5150 & -1.90 \\
\hline 9 & 4994.646 & 5200.5 & 6645.063 & 5000 & -3.85 \\
\hline
\end{tabular}

\begin{tabular}{|c|c|c|c|c|c|}
\hline 10 & 5125.116 & 5200.5 & 6664.84 & 5000 & -6.54 \\
\hline 11 & 5181.657 & 5200.5 & 6679.184 & 5166.67 & -1.59 \\
\hline 12 & 5258.041 & $\begin{array}{l}5381.5 \\
\end{array}$ & 6698.961 & 5166.67 & 1.31 \\
\hline 13 & 5276.928 & 5562.5 & 6830.214 & 5166.67 & 3.33 \\
\hline 14 & 5422.499 & 5562.5 & 6851.811 & 5166.67 & -0.64 \\
\hline 15 & 5564.537 & 5569.5 & 7022.617 & 5680 & -1.22 \\
\hline 16 & 5573.594 & 5562.5 & 7037.891 & 5680 & -2.91 \\
\hline 17 & 5596.97 & 5653 & 7201.444 & 5680 & -0.35 \\
\hline 18 & 5657.125 & 6286.5 & 7229.323 & 5680 & 1.43 \\
\hline 19 & 6367.285 & 6377 & 7266.194 & 5716.67 & -1.44 \\
\hline 20 & 6387.062 & 6648.5 & 7286.012 & 5716.67 & 3.00 \\
\hline 21 & 6600.091 & 6648.5 & 7315.685 & 5775 & -1.28 \\
\hline 22 & 6613.586 & 6648.5 & 7370.513 & 5775 & -1.28 \\
\hline 23 & 6624.397 & 6648.5 & 7482.821 & 5775 & 2.21 \\
\hline 24 & 6645.063 & 6648.5 & 7508.922 & 5775 & -3.75 \\
\hline 25 & 6664.84 & 6648.5 & 7555.648 & 5775 & -8.33 \\
\hline 26 & 6679.184 & 6648.5 & 7611.365 & 5775 & 4.05 \\
\hline 27 & 6698.961 & 6829.5 & 7917.928 & 5775 & 8.96 \\
\hline 28 & 6830.214 & 6829.5 & 7962.859 & 6333.33 & 3.83 \\
\hline 29 & 6851.811 & 7010.5 & 7995.242 & 6333.33 & 1.33 \\
\hline 30 & 7022.617 & 7010.5 & 8006.917 & 6100 & 0.00 \\
\hline 31 & 7037.891 & 7037.891 & 7191.5 & 6100 & 4.27 \\
\hline 32 & 7201.444 & 7201.444 & 7191.5 & 6300 & 2.44 \\
\hline 33 & 7229.323 & 7229.323 & 7191.5 & 6300 & 2.44 \\
\hline 34 & 7266.194 & 7266.194 & 7282 & 6300 & -2.33 \\
\hline 35 & 7286.012 & 7286.012 & 7372.5 & 6300 & -8.03 \\
\hline 36 & 7315.685 & 7315.685 & 7372.5 & 6300 & -3.82 \\
\hline 37 & 7370.513 & 7370.513 & 7553.5 & 6300 & -3.08 \\
\hline 38 & 7482.821 & 7482.821 & 7553.5 & 6160 & 6.21 \\
\hline 39 & 7508.922 & 7508.922 & $\begin{array}{l}7553.5 \\
\end{array}$ & 6160 & -2.99 \\
\hline 40 & 7555.648 & 7555.648 & 7644 & 6160 & -2.99 \\
\hline 41 & 7611.365 & 7611.365 & 7915.5 & 6160 & 0.16 \\
\hline 42 & 7917.928 & 7917.928 & 7915.5 & 6275 & -1.95 \\
\hline 43 & 7962.859 & 7962.859 & $\begin{array}{l}7915.5 \\
\end{array}$ & 6275 & -3.46 \\
\hline 44 & 7995.242 & 7995.242 & 8006 & 6275 & -8.39 \\
\hline 45 & 8006.917 & 8006.917 & 8096.5 & 6625 & -2.57 \\
\hline 46 & 8023.08 & 8023.08 & 8096.5 & 6625 & 3.52 \\
\hline 47 & 8053.627 & 8053.627 & 8096.5 & 6666.67 & 1.78 \\
\hline 48 & 8082.355 & 8082.355 & 8096.5 & 6666.67 & 1.78 \\
\hline 49 & 8096.739 & 8096.739 & 8277.5 & 6666.67 & 0.25 \\
\hline 50 & 8279.262 & 8279.262 & 8368 & 7033.73 & 1.94 \\
\hline 51 & 8361.079 & 8361.079 & 8458.5 & 6866.67 & 0.24 \\
\hline 52 & 8398.855 & 8398.855 & 8458.5 & 6866.67 & -4.63 \\
\hline 53 & 8416.797 & 8416.797 & 8639.5 & 6866.67 & 0.98 \\
\hline 54 & 8586.698 & 8586.698 & 8639.5 & 7040 & 9.15 \\
\hline 55 & 8608.254 & 8608.254 & 8639.5 & 7040 & 7.48 \\
\hline 56 & 8653.201 & 8653.201 & 8911 & 7040 & -3.56 \\
\hline 57 & 8975.038 & 8975.038 & 9182.5 & 6300 & -9.35 \\
\hline 58 & 9235.707 & 9235.707 & 9363.5 & 6300 & -6.67 \\
\hline 59 & 9439.827 & 9439.827 & 9544.5 & $\begin{array}{l}7550 \\
\end{array}$ & 0.00 \\
\hline 60 & 9636.734 & 9636.734 & 9594.5 & 7700 & -1.28 \\
\hline
\end{tabular}

The particular model has to be selected based on maximum number of minimum value of the parameters of residual analysis and average error. The estimated data based on the selected model has to be used to form a knowledge base. It has been observed that ACO has been preferred over all models (table 4) for initial data sets (training data set) using Iris data set. The said concept has also been tallied (table 8) using new data for Iris data set. The modified ACO and other algorithms have been applied on the other data sets like wine data set and Boston city data to produce the estimated data and estimated error. Using Wine data set, it has been observed that out of seven cases modified ACO has been preferred in seven cases (training data table 11) and seven cases in testing data (Table 12). It has been further observed that average error of modified ACO $0.97 \%$ (training data) and $0.55 \%$ (tested data) as compared to conventional ACO $2.33 \%$ (training data) and $2.67 \%$ (tested data). Therefore, modified ACO has been considered as preferable optimizer for tested data with an objective of knowledge extraction of tested antecedent data for Wine data. Using Boston city data set, it has been observed that out of seven cases, modified ACO has been preferred by seven cases in both training (Table 13) and testing data (Table 14) respectively. It has been further observed that average error of modified ACO $0.84 \%$ (training data) and $1.04 \%$ (tested data) as compared to conventional harmony search algorithm $1.97 \%$ (training data) and $1.56 \%$ (tested data).Therefore the modified ACO has been selected 
as the preferable optimizing model for extraction of knowledge for Boston city data. Thereafter for any other new data set, the application of rules as decided by factor analysis and the algorithm of ACO can be applied to from the total effect value (antecedent item) for that.

Table 11

Average Error and Residual Analysis Using Wine Data Set (Training)

\begin{tabular}{|c|c|c|c|r|r|r|r|c|}
\hline $\begin{array}{c}\text { Serial } \\
\text { Number }\end{array}$ & $\begin{array}{c}\text { Method } \\
\text { Name }\end{array}$ & $\begin{array}{c}\text { Sum of } \\
\text { Absolute } \\
\text { Residual }\end{array}$ & $\begin{array}{c}\text { Maximum } \\
\text { Absolute } \\
\text { Residual }\end{array}$ & $\begin{array}{c}\text { Mean } \\
\text { Absolute } \\
\text { Residual }\end{array}$ & $\begin{array}{c}\text { Mean of } \\
\text { Mean } \\
\text { Absolute } \\
\text { Residual }\end{array}$ & $\begin{array}{c}\text { Median } \\
\text { of } \\
\text { Absolute } \\
\text { Residual }\end{array}$ & $\begin{array}{c}\text { Standard } \\
\text { Deviation } \\
\text { of } \\
\text { Absolute } \\
\text { residual }\end{array}$ & $\begin{array}{c}\text { Average } \\
\text { Eror } \\
\text { (\%) }\end{array}$ \\
\hline 1 & $\begin{array}{c}\text { Linear } \\
\text { Equation }\end{array}$ & 563239.8 & 20864.46 & 11.83 & 0.07 & 3715.2 & 2706.837 & 6.65 \\
\hline 2 & $\begin{array}{c}\text { Exponential } \\
\text { equation }\end{array}$ & 263976.8 & 16307.86 & 4.99 & 0.03 & 1216.55 & 1906.527 & 2.8 \\
\hline 3 & $\begin{array}{c}\text { Asymptotic } \\
\text { Equation }\end{array}$ & 916816.2 & 22028.48 & 23.31 & 0.13 & 6067.63 & 3349.786 & 13.1 \\
\hline 4 & $\begin{array}{c}\text { Curvilinear } \\
\text { Equation }\end{array}$ & 272310 & 12907.52 & 5.82 & 0.03 & 363.57 & 1348.284 & 3.27 \\
\hline 5 & $\begin{array}{c}\text { Logarithm } \\
\text { Equation }\end{array}$ & 500072.4 & 20067.69 & 9.84 & 0.06 & 3227.99 & 2545.412 & 5.53 \\
\hline 6 & $\begin{array}{c}\text { Fuzzy } \\
\text { Logic }\end{array}$ & 191872.5 & 6591.45 & 3.89 & 0.02 & 1043.65 & 841.5369 & 2.19 \\
\hline 7 & $\begin{array}{c}\text { Neural } \\
\text { Network }\end{array}$ & 90976.54 & 1019.61 & 1.97 & 0.01 & $\underline{17.65}$ & 298.4702 & 1.11 \\
\hline 8 & GA & 77766.62 & 1019.61 & 1.6 & 0.01 & $\underline{17.65}$ & 288.5529 & 0.9 \\
\hline 9 & PSO & 82189.65 & 1584.68 & 1.85 & 0.01 & $\underline{6.18}$ & 365.586 & 1.03 \\
\hline 10 & ACO & $\underline{82429.31}$ & $\underline{1037.39}$ & $\underline{1.73}$ & $\underline{0.01}$ & $\underline{17.65}$ & $\underline{269.9827}$ & $\underline{0.97}$ \\
\hline
\end{tabular}

Table 12

Average Error and Residual Analysis Using Wine Data Set(Tested)

\begin{tabular}{|c|c|c|c|c|c|c|c|c|}
\hline $\begin{array}{c}\text { Serial } \\
\text { Number }\end{array}$ & $\begin{array}{c}\text { Method } \\
\text { Name }\end{array}$ & $\begin{array}{c}\text { Sum of } \\
\text { Absolute } \\
\text { Residual }\end{array}$ & $\begin{array}{c}\text { Maximum } \\
\text { Absolute } \\
\text { Residual }\end{array}$ & $\begin{array}{c}\text { Mean } \\
\text { Absolute } \\
\text { Residual }\end{array}$ & $\begin{array}{c}\text { Mean of } \\
\text { Mean } \\
\text { Absolute } \\
\text { Residual }\end{array}$ & $\begin{array}{c}\text { Median } \\
\text { of } \\
\text { Absolute } \\
\text { Residual }\end{array}$ & $\begin{array}{c}\text { Standard } \\
\text { Deviation } \\
\text { of } \\
\text { obsolute } \\
\text { residual }\end{array}$ & $\begin{array}{c}\text { Average } \\
\text { Error } \\
(\%)\end{array}$ \\
\hline 1 & $\begin{array}{c}\text { Linear } \\
\text { Equation }\end{array}$ & 242461.8 & 17217.92 & 4.764317 & 0.071109 & 4009.03 & 3294.108 & 7.11092 \\
\hline 2 & $\begin{array}{c}\text { Exponential } \\
\text { equation }\end{array}$ & 121704.4 & 12757.49 & 2.082021 & 0.031075 & 1494.3 & 2341.779 & 3.107494 \\
\hline 3 & $\begin{array}{c}\text { Asymptotic } \\
\text { Equation }\end{array}$ & 359025 & 18334.91 & 9.129402 & 0.13626 & 6385.15 & 3794.556 & 13.62597 \\
\hline 4 & $\begin{array}{c}\text { Curvilinear } \\
\text { Equation }\end{array}$ & 100678.5 & 9395.47 & 2.079314 & 0.031035 & 69.74 & 1615.817 & 3.103454 \\
\hline 5 & $\begin{array}{c}\text { Logarithm } \\
\text { Equation }\end{array}$ & 212114.9 & 16416.51 & 3.91513 & 0.058435 & 3535.42 & 3061.499 & 5.843478 \\
\hline 6 & Fuzzy Logic & 79867.98 & 3048.42 & 1.541123 & 0.023002 & 1029.17 & 798.3215 & 2.300183 \\
\hline 7 & $\begin{array}{c}\text { Neural } \\
\text { Network }\end{array}$ & 19454.74 & 996.42 & 0.386516 & 0.005769 & $\underline{3.17}$ & 233.6207 & 0.576889 \\
\hline 8 & GA & 19068.94 & 996.42 & 0.372862 & 0.005565 & $\underline{3.17}$ & 234.3948 & 0.55651 \\
\hline 9 & PSO & 35301.4 & 2398.57 & 0.7353 & 0.0110 & 8.3 & 526.5675 & 1.094 \\
\hline 10 & ACO & 19056.6 & 996.42 & $\underline{0.372102}$ & $\underline{0.005554}$ & $\underline{3.17}$ & $\underline{234.1222}$ & $\underline{0.555375}$ \\
\hline
\end{tabular}

Table 13

Average Error and Residual Analysis Using Boston City Data (Training)

\begin{tabular}{|c|c|c|c|c|c|c|c|c|}
\hline $\begin{array}{c}\text { Serial } \\
\text { Number }\end{array}$ & $\begin{array}{c}\text { Method } \\
\text { Name }\end{array}$ & $\begin{array}{c}\text { Sum of } \\
\text { Absolute } \\
\text { Residual }\end{array}$ & $\begin{array}{c}\text { Maximum } \\
\text { Absolute } \\
\text { Residual }\end{array}$ & $\begin{array}{c}\text { Mean } \\
\text { Absolute } \\
\text { Residual }\end{array}$ & $\begin{array}{c}\text { Mean of } \\
\text { Mean } \\
\text { Absolute } \\
\text { Residual }\end{array}$ & $\begin{array}{c}\text { Median } \\
\text { of } \\
\text { obsolute } \\
\text { Residual }\end{array}$ & $\begin{array}{c}\text { Standard } \\
\text { Deviation } \\
\text { of } \\
\text { Absolute } \\
\text { residual }\end{array}$ & $\begin{array}{c}\text { Average } \\
\text { Error } \\
(\%)\end{array}$ \\
\hline 1 & $\begin{array}{c}\text { Linear } \\
\text { Equation }\end{array}$ & 2608225 & 30884.83 & 57.81 & 0.11 & 6071.3 & 4104.223 & 11.43 \\
\hline 2 & $\begin{array}{c}\text { Exponential } \\
\text { equation }\end{array}$ & 4386720 & 30783.82 & 78.66 & 0.16 & 10190.87 & 5003.399 & 15.55 \\
\hline 3 & $\begin{array}{c}\text { Asymptotic } \\
\text { Equation }\end{array}$ & 1034558 & 17429.51 & 24.25 & 0.05 & 674.99 & 2149.16 & 4.79 \\
\hline 4 & $\begin{array}{c}\text { Curvilinear } \\
\text { Equation }\end{array}$ & 1250565 & 18522.46 & 27.79 & 0.05 & 118.06 & 2495.727 & 5.49 \\
\hline
\end{tabular}

\begin{tabular}{|c|c|r|r|r|r|r|r|r|}
5 & $\begin{array}{c}\text { Logarithm } \\
\text { Equation }\end{array}$ & 1970980 & 26466.41 & 45.18 & 0.09 & 3671.98 & 3411.724 & 8.93 \\
\hline 6 & $\begin{array}{c}\text { Fuzzy } \\
\text { Logic }\end{array}$ & 635987.1 & 2821.05 & 10.06 & 0.02 & 341.66 & 813.3729 & 1.99 \\
\hline 7 & $\begin{array}{c}\text { Neural } \\
\text { Network }\end{array}$ & 365430.3 & 1408.05 & 6 & 0.01 & 1071.34 & 414.4377 & 1.18 \\
\hline 8 & GA & 330512.2 & 1590.05 & 4.84 & 0.01 & 1071.34 & 411.074 & 0.96 \\
\hline 9 & PSO & 526228.4 & 3402.18 & 6.8 & 0.01 & 101.41 & 976.207 & 1.35 \\
\hline 10 & ACO & $\underline{316549.6}$ & $\underline{5652.02}$ & $\underline{4.3}$ & $\underline{0.01}$ & $\underline{1071.34}$ & 493.7084 & $\underline{0.85}$ \\
\hline
\end{tabular}

Table 14

Average Error and Residual Analysis Using Tested Data on Boston City Data(Tested)

\begin{tabular}{|c|c|c|c|c|c|c|c|c|}
\hline $\begin{array}{c}\text { Serial } \\
\text { Number }\end{array}$ & $\begin{array}{c}\text { Method } \\
\text { Name }\end{array}$ & $\begin{array}{c}\text { Sum of } \\
\text { Absolute } \\
\text { Residual }\end{array}$ & $\begin{array}{c}\text { Maximum } \\
\text { Absolute } \\
\text { Residual }\end{array}$ & $\begin{array}{c}\text { Mean } \\
\text { Absolute } \\
\text { Residual }\end{array}$ & $\begin{array}{c}\text { Mean of } \\
\text { Mean } \\
\text { Absolute } \\
\text { Residual }\end{array}$ & $\begin{array}{c}\text { Median } \\
\text { of } \\
\text { Absolute } \\
\text { Residual }\end{array}$ & $\begin{array}{c}\text { Standard } \\
\text { Deviation } \\
\text { of } \\
\text { Absolute } \\
\text { residual }\end{array}$ & $\begin{array}{c}\text { Average } \\
\text { Error } \\
(\%)\end{array}$ \\
\hline 1 & $\begin{array}{c}\text { Linear } \\
\text { Equation }\end{array}$ & 1116324 & 29669.76 & 36.8 & 0.24 & 5493.49 & 6083.05 & 24.37 \\
\hline 2 & $\begin{array}{c}\text { Exponential } \\
\text { equation }\end{array}$ & 1591262 & 29546.42 & 42 & 0.28 & 9629.36 & 5755.885 & 27.81 \\
\hline 3 & $\begin{array}{c}\text { Asymptotic } \\
\text { Equation }\end{array}$ & 404876.6 & 16309.43 & 15.18 & 0.1 & 3180.23 & 3246.44 & 10.06 \\
\hline 4 & $\begin{array}{c}\text { Curvilinear } \\
\text { Equation }\end{array}$ & 458333.7 & 17380.78 & 16.63 & 0.11 & 3770.79 & 3551.805 & 11.02 \\
\hline 5 & $\begin{array}{c}\text { Logarithm } \\
\text { Equation }\end{array}$ & 841487.6 & 25258.46 & 28.89 & 0.19 & 6333.96 & 5108.946 & 19.13 \\
\hline 6 & $\begin{array}{c}\text { Fuzzy } \\
\text { Logic }\end{array}$ & 198607.2 & 2804.41 & 4.09 & 0.03 & 304.97 & 840.9338 & 2.71 \\
\hline 7 & $\begin{array}{c}\text { Neural } \\
\text { Network }\end{array}$ & 114148.3 & 1391.41 & 2.26 & 0.01 & 1108.03 & 419.2308 & 1.5 \\
\hline 8 & GA & 100868.2 & 1391.41 & 1.77 & 0.01 & 1108.03 & 414.0762 & 1.17 \\
\hline 9 & PSO & 147805.6 & 1791.11 & 1.52 & 0.04 & 803.74 & 932.314 & 1.51 \\
\hline 10 & ACO & 99462.27 & $\underline{2845.43}$ & 1.57 & $\underline{0.01}$ & 1108.03 & 527.8877 & 1.04 \\
\hline
\end{tabular}

From previous knowledge, the necessary consequent item (output) may be decided, which is the predicted value or inference knowledge gathered from the applied early data set. In case of Iris flower data the quality of flower can be inference from the input of its attributes. The quality of wine and property tax rate to be pays by the customer can also be predicted based on the attribute of Wine and Boston city data sets. Thereafter, if the said knowledge is available in advance, necessary planning work can be decided by the Governments and various other agencies in the country.

\section{CONCLUSION}

The applications of rules as decided by factor analysis and principal component analysis have been applied to from the total effect value (antecedent) on the Iris flower data. The methods of statistical analysis and soft computing have been applied on the total effect value to select the preferable method. The decision of the preferable model has been decided by maximum number of minimum parameters of average error and the residual analysis. For checking the validity of model, an effort has been made to get relevant information (knowledge) using new data items (tested data). It has been mentioned that modified harmony search algorithm has been preferred over all models for initial data sets (training data set) using Iris data set. The said concept has been tallied using new data (tested data) for Iris data set.

For any new data set, the said models can be applied to from the cumulative antecedent item for that data set and accordingly a relation can be formed between antecedent item and consequent item for the formation of knowledge base. From previous knowledge, the necessary consequent item may be decided, which is the predicted or inference knowledge gathered from the applied new data set. 
Knowledge discovery (output extraction) can also be made for different new unknown data set.

Thereafter, if the said information is available in advance, necessary planning work can be decided by the Governments and various other agencies in the country

\section{REFERENCES}

[1] Thomas STUTZLE and Marco DORIGO "ACO Algorithms for the Traveling Salesman Problem", John Wiley \& Sons, 1999

[2] Wei Zhao ; Coll. of Inf. Technol., JiLin Agric. Univ., Changchun, China ; Xingsheng Cai ; Ying Lan, "A New Ant Colony Algorithm for Solving Traveling Salesman Problem", Computer Science and Electronics Engineering (ICCSEE), 2012 International Conference, Vol.3, pp. 530-533 23-25 March 2012

[3] Héctor D. Menéndez, Fernando E. B. Otero, David Camacho, "MACOC: A Medoid-Based ACO Clustering Algorithm" 9th International Conference, ANTS 2014, Brussels, Belgium, September 10-12, 2014. Proceedings, pp. 122-133, 2014.

[4] Xiaoyong Liu, "Ant Colony Optimization Algorithm Based on Dynamical Pheromones for Clustering Analysis", International Journal of Hybrid Information Technology, Vol.7, No.2 (2014), pp.29-38

[5] K. Ayari, S. Bouktif, G. Antoniol," Automatic Mutation Test Input Data Generation via Ant Colony," In the Proceedings of the $9^{\text {th }}$ annual conference on Genetic and evolutionary computation [GECCO], London, England, July 2007.

[6] D.J. Mala and V. Mohan, "IntelligenTester - Software Test Sequence Optimization Using Graph Based Intelligent Search Agent," In the Proceedings of International Conference on Computational Intelligence and Multimedia Applications [ICCIMA], 2007, pp. 2227.

[7] K. Li, Z. Yang, "Generating Method of Pair-wise Covering Test Data Based on ACO," In the Proceedings of International Workshop on Educational Technology \& International Workshop on Geoscience and Remote Sensing, 2008, pp. 776-779.

[8] D.J. Mala, M. Kamalapriya, R. Shobhana, V.Mohan, "A NonPheromone based Intelligent Swarm Optimization Technique in Software Test Suite Optimization," In the Proceedings of IAMA, 2009.

[9] P. R. Srivastava, K. Baby, and G Raghurama, "An Approach of Optimal Path Generation using Ant Colony Optimization," In the Proceedings of TENCON 2009, IEEE Press, 2009, pp. 1-6.

[10] K. Li, Z. Zhang, and W. Liu, "Automatic Test Data Generation Based On Ant Colony Optimization," In the Proceedings of Fifth International Conference on Natural Computation (ICNC), IEEE Press, 2009, pp. 216-219.

[11] W. Ding, J. Kou, K. Li, Z. Yang, "An Optimization Method of Test Suite in Regression Test Model," In the Proceedings of the 2009 WRI World Congress on
Software Engineering (WCSE), IEEE Computer Society Washington, DC, USA, Vol. 4, 2009, pp. 180-183.

[12] M. Chis, "A Survey of the Evolutionary Computation Techniques for Software Engineering," 1st Chapter In Chis, M. (Ed.), Evolutionary Computation and Optimization Algorithms in Software Engineering: Applications and Techniques, 2010, pp. 1-12.

[13] R. F. Tavares Neto and M. Godinho Filho, "Literature review regarding Ant Colony Optimization applied to scheduling problems: Guidelines for implementation and directions for future research", Engineering Applications of Artificial Intelligence, Volume 26 Issue 1, pp 150-161. January, 2013

[14] D. P. Singh, J. P. Choudhury and M. De, “ A Comparative Study on the performance of Soft Computing models in the domain of Data Mining," International Journal of Advancements in Computer Science and Information Technology, Vol. 1, No. 1, pp. 35-49, September, 2011

[15] D. P. Singh, J. P. Choudhury and M. De, "A Comparative Study to Select a Soft Computing Model for Developing the Knowledge Base of Data mining with Association Rule Formation by Factor Analysis", International Journal of Artificial Intelligence and Knowledge Discovery, Vol. 3, No. 3, October, 2013

[16] D. P. Singh, J. P. Choudhury and M. De, "A comparative study on the performance of Fuzzy Logic, Bayesian Logic and neural network towards Decision Making"International Journal of Data Analysis Techniques and Strategies (IJDATS), Vol. 4, No. 2, pp. 205-216, April, 2012

[17] D. P. Singh, J. P. Choudhury and M. De, "A Comparative Study to Select a Soft Computing Model for Knowledge Discovery in Data Mining”, International Journal of Artificial Intelligence and Knowledge Discovery, Vol. 2, No. 2, pp. 6-19, April, 2012.

[18] D. P. Singh, J. P. Choudhury and M. De, "A comparative study on principal component analysis a $\mathrm{nd}$ factor analysis for the formation of association rule in datamining domain", Proceedings of the $2^{\text {nd }}$ International Conference on Mathematical, Computational and Statistical Sciences (MCSS '14), Gdansk, Poland, ISBN: 978-960-474-380-3, pp.442-452, May 15-17, 2014, ISI Index

\section{AUTHOR PROFILE}

Dr. Dharmpal Singh received his Bachelor of Computer Science and Engineering and Master of Computer Science and Engineering from West Bengal University of Technology. He has about eight years of experience in teaching and research. At present, he is with Pailan College of Management and Technology, Kolkata, and West Bengal, India as an Associate Professor. Currently, he had done his $\mathrm{Ph}$. D in University of Kalyani. He has about 23 publications in national and international journals and conference proceedings. 
Prof. (Dr.) Jagannibas Paul Choudhury received his Bachelor of Electronics and Tele-Communication Engineering with Honours from Jadavpur University, Kolkata and Masters of Technology from Indian Institute of Technology Kharagpur. He received his $\mathrm{PhD}$ (Engineering) from Jadavpur University. He has about 24 years experience in teaching, research and administration. Now, he is with Department of Information Technology, Kalyani Government Engineering College, West Bengal, India as an Associate Professor and the Head in Information Technology. He has about 100 publications in national and international journals and conference proceedings. His research fields are soft computing, data mining, clustering and classification, routing a computer network, etc.

Prof. (Dr.) Mallika De received her BSc in Physics from Calcutta University in 1973 and MSc from Jadavpur University in 1976. She received MTech in Computer Science from Indian Statistical Institute, Calcutta in 1980 and 1985, respectively, and $\mathrm{PhD}$ in Engineering from Jadavpur University in 1997. She is currently JIS Group, India; she has 30 years as a faculty. Her research interests are parallel algorithms and architectures, fault-tolerant computing, image processing and soft computing. She has authored of ten refereed journal articles and ten conference papers. 